\title{
Current status and future of delivery systems for prevention and treatment of infections in the oral cavity
}

Sevda Şenel ( $\sim$ ssenel@hacettepe.edu.tr)

Hacettepe Universitesi Eczacilik Fakultesi

Ayben Işılay Özdoğan

Turkish Patent and Trademark Office

Gülçin Akca

Gazi University

\section{Research Article}

Keywords: oral infections, topical drug delivery, nanoparticulate systems, hydrogels, fibers, strips, new therapeutic agents

Posted Date: February 9th, 2021

DOI: https://doi.org/10.21203/rs.3.rs-196692/v1

License: (c) (i) This work is licensed under a Creative Commons Attribution 4.0 International License. Read Full License

Version of Record: A version of this preprint was published at Drug Delivery and Translational Research on March 26th, 2021. See the published version at https://doi.org/10.1007/s13346-021-00961-2. 


\section{Abstract}

Oral health reflects the general health and it is fundamental to well-being and quality of life. An infection in the oral cavity can be associated with serious complications in human health. Local therapy of these infections offers many advantages over systemic drug administration, targeting directly to the diseased area while minimizing systemic side effects. Specialized drug delivery systems into the oral cavity have to be designed in such a fashion that they resist to the aqueous environment that is constantly bathed in saliva and subject to mechanical forces. Additionally, a prolonged release of drug should also be provided, which would enhance the efficacy and also decrease the repeated dosing. This review is aimed to summarize the current most relevant findings related to local drug delivery of various drug groups for prevention and treatment of infections (viral, bacterial, fungal) and infection related manifestations in the oral cavity. Current therapeutic challenges in regard to effective local drug delivery systems will be discussed and the recent approaches to overcome these obstacles will be reviewed. Finally, future prospects will be overviewed to promote novel strategies that can be implemented in clinical management for prevention and treatment of oral infections.

\section{Introduction}

The oral cavity, which is the main entrance for two systems vital to human function and physiology, the gastrointestinal and respiratory systems, consists of the teeth, the buccal, sublingual and gingival mucosa, soft and hard palate and tongue (Fig. 1). It has a very large and diverse microbiota, harboring numerous microorganisms which include bacteria, fungi, viruses and protozoa. There is a homeostasis between this microbiota and the host, which forms an environment with specific dynamics, and plays a crucial role in maintaining the oral health as well as the systemic health. Disruption of this balance by various factors will result in dysbiosis, allowing for the survival and establishment of a more virulent polymicrobial community impairing the efficient immune responses. Subsequently, these events can clinically manifest as oral infectious diseases [1]. For the pathogenesis of the oral infections, not only the microbiological aspects but also the immunological host response needs to be considered as crucial elements.

Oral diseases pose a major health burden for many countries and affect people throughout their lifetime, causing pain, discomfort, disfigurement and even death. These diseases share common risk factors with other major noncommunicable diseases. It is estimated by the World Health Organisation (WHO) that oral diseases affect nearly 3.5 billion people [2]. Oral health is considered as a key indicator of overall health, well-being and quality of life. In 2019, oral health has been included in the Political Declaration on Universal Health Coverage of United Nations Political Declaration [3]. Several systemic diseases manifest in the oral cavity. Vice versa, specific conditions in the oral cavity may create foci of infection that can affect many other vital systems, such as the cardiovascular and renal systems.

\section{Virus related oral infections}

Oral infections, based on their microbial etiology, can be reviewed in three essential groups, which are viral, fungal and bacterial. Although, frequency of oral viral infections is not very high as the bacterial infections and exerts diagnostic challenges, it may be linked to some severe results in the oral cavity [4-6].

Many dormant viruses that are present in the human oral cavity can be activated and produce a variety of pathological changes in the oral mucosa in individuals immunocompromised by age, illness, or as a side effect of therapy (e.g. anticancer therapy) [7]. Oral manifestations of general viral infections may be presented as a primary sign of disease, co-symptom of the disease, or the only sign observed in such viral disease. Among the major types of viruses that are responsible for oral infections are Paramyxoviridae, Coxsackieviruses (a subgroup of the RNA enteroviruses), oral papillomas (human Papillomavirus of the Papovavirus family) and human herpesvirus family (Fig. 2). HPV infections have received particular attention in recent years, as persistent strains might increase the risk of experiencing malignant transformation in the oral mucosa. Many other viral infections can affect the oral cavity in humans, either as localized or systemic infections [8, 9]. Diagnosis and early management of viral infections is very critical, as certain viral infections result in serious conditions [4]. Recurrent Herpes Labialis (RHL) is a commonly occurring condition in herpes simplex virus (HSV) infection, characterized as a lesion located on the lips and occasionally the attached gingiva (known as a fever blister or cold sore). Secondary infections from oral bacteria can prolong the healing process. Recurrent intraoral herpes $(\mathrm{RIH})$, which is observed more often in immunocompromised patients, may be difficult to distinguish clinically from other oral mucosal disorders, such as aphthous stomatitis [10]. Topical therapies for oral HSV infections can be categorized as palliative, preventive and antiviral. Among the palliative topical agents are anesthetics such as benzocaine and lidocaine which are helpful in reducing pain associated with an oral HSV infection. Recurrent herpetic lesions are usually treated with topical antiviral medications such as acyclovir and penciclovir [11]. In immunocompetent patients, oral or parenteral administration has been shown to be more effective [4]. Currently, available antiviral medications or treatments are limited and their efficacy is inadequate.

Severe acute respiratory syndrome coronavirus 2 (SARS-CoV-2), which is a highly transmissible and pathogenic coronavirus has emerged in late 2019, and it causes acute respiratory disease, named 'coronavirus disease 2019' (COVID-19), which threatens human health and public safety. On March 2020, WHO has defined COVID-19 as pandemic [12]. At the time this paper was submitted (January 2020), more than 90 million cases and more than 1.9 million deaths have been reported, with an enormously rapid day-by-day increase in these numbers due to mutations in the virus. SARS-CoV-2 is transmitted from human-tohuman by either direct transmission such as cough, sneeze, and droplet inhalation or contact transmission like saliva, contact through mucous membranes of the mouth, nose, and eyes. SARS-CoV-2 uses ACE2 as the receptor and human proteases as entry activators, subsequently it fuses the viral membrane with the cell membrane and achieves invasion $[13,14]$. ACE-2 expression highly occurs in the oral mucosa epithelium, and the expression is more in the dorsum of the tongue [15]. Wang et al. [16] have reported that SARS-CoV-2 might induce acute sialadenitis and associated symptoms, such as pain, discomfort, inflammation, and secretory dysfunction in salivary glands by fusing with them, replicating and lysing the cells. As oral mucosa is the first area infected with SARS- CoV-2 through droplets [17], oral mucosal lesions, painful ulcers, or blisters could be signs of COVID-19, and they should be examined thoroughly [18]. Currently, there is a low certainty of evidence regarding cause-effect relationship between coronavirus infection and the appearance of oral lesions, however, similar to that of HIV infection, COVID-19 patients were reported to develop oral lesions related to immunosuppression. Taste alteration is the most prevalent

Page 2/31 
reported oral manifestation. The multiple clinical aspects suggest coinfections, immunity impairment, and adverse reactions rather than a genuine oral mucosa infection primarily caused by SARS-CoV-2 [19]. Lack of oral hygiene, opportunistic infections, stress, immunosuppression, vasculitis and hyperinflammatory response secondary to COVID-19 have been reported as the most important predisposing factors for onset of oral lesions in COVID-19 patients. Among the most common oral manifestations of COVID-19 disease, oral dryness, vesiculobullous lesions, aphthous-like lesions, herpetiform lesions, candidiasis, and oral lesions of Kawasaki-like disease have been reported [18, 20-23]. Currently, there are no specific medications for COVID-19 related oral manifestations, but progress in investigations is indeed going faster than usual, because it is considered as a "Once-in-a-Century Pandemic", hence, there would be new effective treatments available for clinical applications in coming months.

\section{Fungi related oral infections}

Candidal and non-candidal fungal infections in oral mucosa occur generally as a result of defects in the immune system. Candidiasis (candidosis) is the most common fungal infection of the oral cavity, whilst the incidence of noncandidal oral fungal infections such as aspergillosis, cryptococcosis, histoplasmosis, blastomycosis, paracoccidioidomycosis, zygomycosis (mucormycosis), oral geotrichosis, Rhodotorula infection, and fusariosis is rather low [24]. The most commonly seen fungal oral infections are summarized in Fig. 1. Fungal infections can be superficial, may cause serious lesions in the oral cavity or can be indicative of a more serious systemic illness, that may even result in mortality [25].

Various systemic and topical agents are used in treatment of candidiasis. Topical delivery has been preferred predominantly in uncomplicated cases, whilst systemic delivery is indicated when topical agents are ineffective or not tolerated in cases such as immunocompromised HIV or patients with cancer. Nystatin, amphotericin B, miconazole, ketoconazole, clotrimazole are the most commonly used topical antifungal drugs. Other systemic (oral or parenteral) treatment alternatives such as itraconazole, voriconazole, posaconazole, anidulafungin, caspofungin and isavuconazole have also found applications in treatment [2628].

\section{Bacteria related oral infections}

The oral cavity harbors more than 700 different bacterial species [29]. Oral bacterial infections can occur with intense clinical symptoms, chronic or without apparent symptoms or clinical findings without impairing the host defenses like disrupting the mucosal barriers. Oral infections commonly originate from an odontogenic (tooth) source in adults and from tonsil and lymphatic sources in children. Odontogenic infections arise from advanced dental caries or periodontal disease. Nonodontogenic oral infections are related to salivary gland infection, lymph node abscess, postoperative infection, chemical, thermal, or trauma injury, and may be associated with almost any microorganism. Sexually transmitted pathogens such as herpes simplex, Neisseria gonorrhea, and Treponema pallidum may be considered [30]. These nonodontogenic infections can be potentially life threatening.

The oral mucosal infections can be widespread (stomatitis/mucositis, glossitis, gingivitis) or localized (white lesions, red lesions, ulcers) [31]. The clinical manifestations and bacteria responsible for oral infections are summarized in Fig. 2. Complex biofilms of varying compositions of bacteria can colonize the surfaces of the oral cavity. Dental plaque is the term commonly used for the biofilm formed on teeth, however, this term has now been extended to include biofilms on all oral surfaces. These biofilms consist of complex microbial communities embedded in a matrix of polymers of bacterial and salivary origin and they are recognized as a virulence factor in many oral infectious diseases, including dental caries, periodontitis and endodontic infections [32, 33]. Periodontitis, which is a chronic inflammatory disease, may result in progressive destruction of the periodontal ligament and alveolar bone with periodontal pocket formation, gingival recession or both. Periodontitis can also affect many other vital systems, such as the cardiovascular and renal [34].

On the other hand, severe periodontal inflammation or bleeding may require careful investigation of conditions such as diabetes mellitus, human immunodeficiency virus infection, thrombocytopenia, and leukemia [35]. It is important to diagnose correctly the underlying local or systemic condition of the oral diseases for the right treatment. Examination of the oral cavity should include evaluation for mucosal changes, periodontal inflammation and bleeding, and general condition of the teeth. Oral manifestations of specific systemic conditions are oral lesions (including ulcerative, erosive, or white lesions); swelling; erythema, mucosal pallor and atrophy, change in mucosal pigmentation, periodontal bleeding and inflammation [35, 36].

Antibacterial agents such as chlorhexidine, metronidazole and tetracycline are used topically in the management of these infections, hence higher concentration of the antibiotic can be available in the affected area and a much lower concentration throughout the rest of the body. By this means, the systemic side effects, as well as the risk of bacterial resistance is decreased. Furthermore, antibacterial agents have been shown to be effective in the disruption/inhibition of oral biofilm. Nevertheless, current antimicrobial treatments have been reported to treat the problem only provisionally and are not effective at complete elimination of the infections, and the challenge of precisely and continuously eliminating the specific pathogens without disturbing the microbial ecology still exists. Alternate strategies against biofilms such as biofilm-inhibition agents, to prevent the early stages of biofilm formation, or biofilmdispersal agents to disrupt the biofilm cell community have not been sufficiently efficient in direct treatment and eradication of the established biofilms [37]. Hence, investigation of alternative agents to antibiotics as well as new delivery systems play a key role to improve the efficacy of the antibacterial therapy against oral infections, without necessarily inducing microbial dysbiosis of the oral cavity. In following sections, such approaches will be reviewed with current examples.

\section{Topical Drug Delivery For Treatment Of Oral Infections}

Topical drug delivery plays an important role in the management of oral infections. Topical drugs have been extensively used as the first line of therapy in many conditions related to viral, bacterial and fungal infections. A large number of clinical studies have established the clinical efficacy of topical antimicrobials and antivirals which provides targeted drug delivery options for the treatment of local oral lesions (see Tables 1-3) [38]. In general, when compared to systemic delivery, topical drug delivery has a number of advantages such as the ability to deliver drug more selectively to a specific site at higher concentrations, lowering risk of systemic adverse events, avoiding fluctuations in drug levels, inter- and intra-patient variations, and suitability for selfmedication, hence improved patient compliance. The other advantages of topical delivery will be mentioned where appropriate. Nonetheless, there are also 
some obstacles faced with topical drug delivery into oral cavity such as taste alterations, limited surface area, poor tissue penetration and rapid removal due to continuous saliva flow and tongue movement and accidental swallowing. The total surface area of the oral mucosa is relatively small $\left(\sim 200 \mathrm{~cm}^{2}\right)$. The teeth, keratinized epithelium, and non-keratinized epithelium occupy about $20 \%, 50 \%$, and $30 \%$ of the total surface area, respectively. The average volumes of saliva present in the mouth before and after swallowing was estimated to be 0.77 and $1.07 \mathrm{~mL}$, respectively, and the average thickness of the salivary film in the mouth was calculated as 0.07 and $0.10 \mathrm{~mm}$ [39]. The permeability to the topical drugs differs significantly in different oral regions, depending on the pattern of epithelial differentiation, such as thickness and the extent of keratinization. Buccal and sublingual regions in the oral cavity are lined by nonkeratinized, stratified squamous epithelium, which is $100-200 \mu \mathrm{m}$ and $8-12$ cells thick in the sublingual region, and $500-800 \mu \mathrm{m}$ and $40-50$ cells thick in the buccal region. The loss of the permeability barrier in the oral mucosa, which can be encountered due to oral manifestations, may lead to rapid diffusion of the drug into tissues when compared to the intact mucosa. The oral epithelium is covered by a complex mucus layer with an average thickness of $70-100 \mu m$, which has an impact on the mobility of delivery systems and drug molecules. Mucus forms a protective coating on epithelial surfaces and plays a key role in host defense. Mucins are the primary structural components of mucus that creates its viscoelastic properties as well as its protecting functions in oral diseases such as HIV/AIDS, oral candidiasis, and dental caries [40]. 
Table 1

Drug delivery systems for treatment of bacterial infection-related conditions in the oral cavity*

\begin{tabular}{|c|c|c|c|c|c|c|}
\hline Drug & Target & Delivery system & Ingredients & In vitro studies & In vivo studies & Results \\
\hline \multicolumn{7}{|l|}{ Antimicrobials } \\
\hline $\begin{array}{l}\text { Ampicillin and } \\
\text { metronidazole }\end{array}$ & Oral mucosa & Fiber & Polylactide & $\begin{array}{l}\text { - Antibacterial } \\
\text { activity agar } \\
\text { diffusion assay } \\
\text { - } \\
\text { Cytocompatibility } \\
\text { human gingival } \\
\text { fibroblasts }\end{array}$ & - & $\begin{array}{l}\text { - Antibacterial effec } \\
\text { against } A \text {. } \\
\text { actinomycetemcom } \\
\text { F. nucleatum, P. gin! } \\
\text { and E. Faecalis } \\
\text { - No cytotoxic effec }\end{array}$ \\
\hline $\begin{array}{l}\text { Cefuroxime } \\
\text { axetil }\end{array}$ & Oral mucosa & $\begin{array}{l}\text { Mono and bilayered } \\
\text { film and wafer }\end{array}$ & Chitosan and HPMC & $\begin{array}{l}\text { - Drug release } \\
\text { Franz diffusion } \\
\text { cells } \\
\text { - Antimicrobial } \\
\text { activity } \\
\text { agar disk } \\
\text { diffusion method }\end{array}$ & - & $\begin{array}{l}\text { - Prolonged release } \\
\text { adhesive chitosan } \mathrm{k} \\
\text { layer and HPMC ba: } \\
\text { drug loaded layer w } \\
\text { suitable mucoadhe: } \\
\text { - Increased antimicr } \\
\text { activity against E. C } \\
\text { S. aureus }\end{array}$ \\
\hline $\begin{array}{l}\text { Chlorhexidine } \\
\text { digluconate }\end{array}$ & $\begin{array}{l}\text { Oral mucosa } \\
\text { Periodontal pocket }\end{array}$ & Film, gel & $\begin{array}{l}\text { Chitosan, TPP, glycerin, } \\
\text { lactic acid }\end{array}$ & $\begin{array}{l}\text { - Mucoadhesion } \\
\text { Texture analyzer } \\
\text { (porcine buccal } \\
\text { mucosa) } \\
\text { - Antimicrobial } \\
\text { activity } \\
\text { blood agar plates }\end{array}$ & - & $\begin{array}{l}\text { - Suitable mucoadh } \\
\text { - Enhanced antimicl } \\
\text { activity against } \\
\text { Porphyromonas gir } \\
\text { in presence of chito }\end{array}$ \\
\hline Chlorhexidine & Oral cavity & Mouthwash & Chitosan & - & $\begin{array}{l}\text { Healthy } \\
\text { volunteers } \\
\text { - plaque index, } \\
\text { gingival index } \\
\text { Quickley-Hein } \\
\text { plaque index } \\
\text { (QPI), probing } \\
\text { depth } \\
\text {-Antimicrobial } \\
\text { activity on dental } \\
\text { plaques } \\
\text { Agar diffusion }\end{array}$ & $\begin{array}{l}\text { - Significant reducti } \\
\text { clinical parameters } \\
\text { presence of chitosa } \\
\text { - Enhanced antimicı } \\
\text { effect against } S . m \iota \\
\text { or enterococci in prt } \\
\text { of chitosan - }\end{array}$ \\
\hline Chlorhexidine & Tooth surface & Varnish & $\begin{array}{l}\text { - Ethyl cellulose and } \\
\text { poly ethylene glycol in } \\
\text { ethanol }\end{array}$ & - & $\begin{array}{l}\text { Orthodontic } \\
\text { patients }(10-16 \\
\text { year-old) } \\
\text { - Antimicrobial } \\
\text { activity in } \\
\text { sputum samples } \\
\text { of orthodontic } \\
\text { patients }\end{array}$ & $\begin{array}{l}\text { - A significant decre } \\
\text { S mutans levels for } \\
\text { weeks } \\
\text { - No significant cha } \\
\text { Actinomyces viscos } \\
\text { levels }\end{array}$ \\
\hline $\begin{array}{l}\text { Chlorhexidine / } \\
\text { thymol }\end{array}$ & Tooth root surface & Varnish & $\begin{array}{l}\text { Vinyl acetate co- } \\
\text { polymer and acrylate } \\
\text { co-polymer }\end{array}$ & $\begin{array}{l}\text { - Antibacterial } \\
\text { activity (agar } \\
\text { difussion assay) }\end{array}$ & $\begin{array}{l}\text { - Patients ( } 35 \\
\text { and } 55 \text { year-old) } \\
\text { with one tooth } \\
\text { with buccal } \\
\text { gingival } \\
\text { recession of } 1- \\
2 \mathrm{~mm} \text { and initial } \\
\text { root caries } \\
\text { (between) }\end{array}$ & $\begin{array}{l}\text { - Significant reducti } \\
\text { Streptococci and } \\
\text { Lactobacilli in } \\
\text { supragingival plaqu } \\
\text { - Stronger antibacte } \\
\text { activity against } \\
\text { A.actinomycetemcc } \\
\text { with ethyl acetate } \mathrm{W} \\
\text { compared to ethanc } \\
\text { solvent } \\
\text { - Highest activity ag } \\
\text { strains: P. gingivalis } \\
\text { Fusobacterium Nuc }\end{array}$ \\
\hline
\end{tabular}




\begin{tabular}{|c|c|c|c|c|c|c|}
\hline Drug & Target & Delivery system & Ingredients & In vitro studies & In vivo studies & Results \\
\hline $\begin{array}{l}\text { Chlorhexidine } \\
\text { and diclofenac } \\
\text { sodium }\end{array}$ & Buccal mucosa & Film & $\begin{array}{l}\text { HPMC, PEG } 400 \text { and } \\
\text { Carbopol } 917\end{array}$ & $\begin{array}{l}\text { - Anti- } \\
\text { inflammatory } \\
\text { activity }\end{array}$ & - & $\begin{array}{l}\text { - Anti-inflammatory } \\
\text { activity by reducing } \\
\text { prostaglandin E2 le' }\end{array}$ \\
\hline $\begin{array}{l}\text { Chlorhexidine } \\
\text { and } \\
\text { Betamethasone }\end{array}$ & & & & $\begin{array}{l}\text { prostaglandin E2 } \\
\text { levels }\end{array}$ & & $\begin{array}{l}\text { - Antibacterial activi } \\
\text { against planktonic } \\
\text { biofilm bacteria }\end{array}$ \\
\hline \multirow[t]{3}{*}{$\begin{array}{l}\text { Chlorhexidine } \\
\text { and Lidocaine }\end{array}$} & & & & & & \multirow[t]{3}{*}{ - No cytotoxic effec } \\
\hline & & & & $\begin{array}{l}\text { - Antibacterial } \\
\text { activity }\end{array}$ & & \\
\hline & & & & $\begin{array}{l}\text { - Cytotoxicity test } \\
\text { HaCaT } \\
\text { keratinocyte cell } \\
\text { line }\end{array}$ & & \\
\hline \multirow[t]{5}{*}{ Doxycycline } & \multirow[t]{5}{*}{ Periodontal pocket } & \multirow{5}{*}{$\begin{array}{l}\text { Nanoparticle } \\
\text { loaded gel }\end{array}$} & \multirow{5}{*}{$\begin{array}{l}\text { Nanoparticles: } \\
\text { chitosan } \\
\text { Gel: PVA, } \\
\text { PVP, } \\
\text { glycerol and } \\
\text { PEG } 400\end{array}$} & \multirow[t]{5}{*}{-} & \multirow{5}{*}{$\begin{array}{l}\text { - Patients with } \\
\text { moderate } \\
\text { chronic } \\
\text { periodontitis } \\
\text { - IL-6 and TNF-a } \\
\text { levels in gingival } \\
\text { crevicular fluid }\end{array}$} & \multirow{5}{*}{$\begin{array}{l}\text { - Reduced probing } \mathrm{F} \\
\text { depth } \\
\text { - Decreased levels o } \\
\text { and TNF-a }\end{array}$} \\
\hline & & & & & & \\
\hline & & & & & & \\
\hline & & & & & & \\
\hline & & & & & & \\
\hline \multirow[t]{3}{*}{ Doxycycline } & \multirow[t]{3}{*}{$\begin{array}{l}\text { Subgingival } \\
\text { placement }\end{array}$} & \multirow[t]{3}{*}{ Strip } & \multirow[t]{3}{*}{ Methylcellulose } & \multirow[t]{3}{*}{-} & $\begin{array}{l}\text { - Patients with } \\
\text { inflammatory } \\
\text { periodontal } \\
\text { disease }\end{array}$ & \multirow{3}{*}{$\begin{array}{l}\text { - Significant decrea: } \\
\text { clinical parameters } \\
\text { week } 8 \\
\text { - Marked decrease i } \\
\text { anaerobic count by } \\
10\end{array}$} \\
\hline & & & & & $\begin{array}{l}\text { - Gingival index, } \\
\text { probing depth, } \\
\text { attachment loss, } \\
\text { and gingival } \\
\text { shrinkage }\end{array}$ & \\
\hline & & & & & $\begin{array}{l}\text { - Microbiological } \\
\text { evaluation in } \\
\text { subgingival fluid }\end{array}$ & \\
\hline \multirow[t]{4}{*}{ Metronidazole } & \multirow[t]{4}{*}{ Periodontal pocket } & \multirow{4}{*}{$\begin{array}{l}\text { Microcapsuleloaded } \\
\text { hydrogel }\end{array}$} & \multirow[t]{4}{*}{ Chitosan, PVA } & - Drug release & \multirow{4}{*}{$\begin{array}{l}\text { Ligation induced } \\
\text { periodontitis in } \\
\text { Wistar rats }\end{array}$} & - Prolonged drug rel \\
\hline & & & & $\begin{array}{l}\text { dialysis diffusion } \\
\text { method }\end{array}$ & & $\begin{array}{l}\text { - Prolonged in vitro } \\
\text { antibacterial activit! }\end{array}$ \\
\hline & & & & $\begin{array}{l}\text { - Bacteriostasis } \\
\text { activitiy }\end{array}$ & & $\begin{array}{l}\text { - Enhanced in vivo } \\
\text { antibacterial activit! }\end{array}$ \\
\hline & & & & & & $\begin{array}{l}\text { - Reduced probing } c \\
\text { of the periodontal } p\end{array}$ \\
\hline \multirow[t]{4}{*}{ Metronidazole } & \multirow[t]{4}{*}{ Periodontal pocket } & \multirow[t]{4}{*}{ Fiber } & \multirow[t]{4}{*}{ Polylactide } & - Drug release & \multirow[t]{4}{*}{-} & $\begin{array}{l}\text { - Prolonged drug rel } \\
\text { after day } 3\end{array}$ \\
\hline & & & & $\begin{array}{l}\text { (immersing fiber } \\
\text { in liquid medium) }\end{array}$ & & - Antibacterial activi \\
\hline & & & & $\begin{array}{l}\text { - Antibacterial } \\
\text { activity agar } \\
\text { diffusion method }\end{array}$ & & $\begin{array}{l}\text { actinomycetemcom } \\
\text { and } P \text {. gingivalis }\end{array}$ \\
\hline & & & & $\begin{array}{l}\text { - Cytotoxicity } \\
\text { human gingival } \\
\text { fibroblasts }\end{array}$ & & \\
\hline \multirow[t]{2}{*}{ Metronidazole } & \multirow[t]{2}{*}{ Periodontal pocket } & \multirow[t]{2}{*}{ Gel } & \multirow[t]{2}{*}{ Chitosan, lactic acid } & \multirow[t]{2}{*}{-} & $\begin{array}{l}\text { Patients with } \\
\text { moderate to } \\
\text { severe chronic } \\
\text { periodontitis }\end{array}$ & $\begin{array}{l}\text { - Significant decrea: } \\
\text { clinical parameters } \\
\text { similar to that of a } \\
\text { commercial gel }\end{array}$ \\
\hline & & & & & $\begin{array}{l}\text { - gingival } \\
\text { recession, plaque } \\
\text { index, gingival } \\
\text { index, and } \\
\text { gingival bleeding } \\
\text { time }\end{array}$ & \\
\hline
\end{tabular}




\begin{tabular}{|c|c|c|c|c|c|c|}
\hline Drug & Target & Delivery system & Ingredients & In vitro studies & In vivo studies & Results \\
\hline $\begin{array}{l}\text { Metronidazole } \\
\text { and } \\
\text { levofloxacin }\end{array}$ & Periodontal pocket & Film & Chitosan & $\begin{array}{l}\text { - Drug release } \\
\text { placing films in } \\
\text { vial containing } \\
\text { Mcllvaine buffer, } \\
\text { pH 6.6) } \\
\text { - Antibacterial } \\
\text { activity disc } \\
\text { diffusion method }\end{array}$ & $\begin{array}{l}\text { Patients with } \\
\text { chronic } \\
\text { periodontitis } \\
\text { - Gingival index, } \\
\text { plaque index and } \\
\text { pocket depth }\end{array}$ & $\begin{array}{l}\text { - Prolonged drug rel } \\
\text { - Significant decrea: } \\
\text { clinical parameters } \\
\text { - Antibacterial activi } \\
\text { against } S \text {. aureus a } \\
\text { coli }\end{array}$ \\
\hline Minocycline & Periodontal pocket & $\begin{array}{l}\text { In-situ forming } \\
\text { cubic liquid crystal }\end{array}$ & $\begin{array}{l}\text { Phytantriol / propylene } \\
\text { glycol }\end{array}$ & $\begin{array}{l}\text { - Drug release } \\
\text { dialysis } \\
\text { membrane } \\
\text { diffusion method }\end{array}$ & $\begin{array}{l}\text { Ligation induced } \\
\text { periodontitis in } \\
\text { SPF rats }\end{array}$ & $\begin{array}{l}\text { - Sustained release } \\
\text { four days } \\
\text { - Reduction in gingi } \\
\text { index, probing deptl } \\
\text { alveolar bone loss }\end{array}$ \\
\hline Minocycline & Periodontal pocket & Liposome & $\begin{array}{l}\text { Hydrogenated soy } \\
\text { phosphatidylcholine } \\
\text { and cholesterol }\end{array}$ & $\begin{array}{l}\text { - Cell proliferation } \\
\text { rate MTT assay } \\
\text { murine } \\
\text { macrophages } \\
\text { (ANA-1) }\end{array}$ & - & $\begin{array}{l}\text { - Inhibition of the } \\
\text { proliferation of } \\
\text { macrophages } \\
\text { - Stronger anti- } \\
\text { inflammatory effect } \\
\text { suppression of TNF } \\
\text { mRNA expression }\end{array}$ \\
\hline Minocycline & Periodontal pocket & Strip & Polycaprolactone & - & $\begin{array}{l}\text { Patients with } \\
\text { chronic } \\
\text { periodontitis } \\
\text { - Subgingival } \\
\text { plaque bacterial } \\
\text { counts on day } 3 \\
\text { (strips inserted in } \\
\text { periodontal } \\
\text { pocket) }\end{array}$ & $\begin{array}{l}\text { Significant reductio } \\
\text { proportions of } C . g r \\
P . \text { melaninogenica, } \\
\text { necrogenes by day }\end{array}$ \\
\hline Moxifloxacin & Periodontal pocket & $\begin{array}{l}\text { Nanoparticle } \\
\text { loaded in situ gel }\end{array}$ & $\begin{array}{l}\text { Nanoparticles: } \\
\text { PLGA, PVA } \\
\text { Gel: } \\
\text { Poloxamer } 407\end{array}$ & $\begin{array}{l}\text { - Drug release } \\
\text { dialysis diffusion } \\
\text { method }\end{array}$ & $\begin{array}{l}\text { - Ligation } \\
\text { induced } \\
\text { periodontitis in } \\
\text { Sprague-Dawley } \\
\text { rats } \\
\text { - y-scintigraphy } \\
\text { analysis in } \\
\text { rabbits }\end{array}$ & $\begin{array}{l}\text { - Extended drug rele } \\
\text { and enhanced reten } \\
\text { the system } \\
\text { - Higher efficacy wit } \\
\text { a-week application } \\
\text { compared to that of } \\
\text { a-day application of } \\
\text { commercial gel } \\
\text { - Almost complete } \\
\text { recovery in } 3 \text { weeks }\end{array}$ \\
\hline Moxifloxacin & Periodontal pocket & In situ gel & $\begin{array}{l}\text { Poloxamer 407, Gellan } \\
\text { gum, Carbopol 934P }\end{array}$ & $\begin{array}{l}\text { - Drug release } \\
\text { Franz diffusion } \\
\text { cell } \\
\text { - The } \\
\text { antibacterial } \\
\text { activity using } \\
\text { agar cup method }\end{array}$ & - & $\begin{array}{l}\text { - Prolonged drug rel } \\
(9 \mathrm{~h}) \\
\text { - Antimicrobial activ } \\
\text { against } S \text {. aureus al } \\
\text { coli in gel }\end{array}$ \\
\hline Moxifloxacin & Oral cavity & Gel & $\begin{array}{l}\text { Chitosan, Carbopol 940, } \\
\text { HPMC }\end{array}$ & $\begin{array}{l}\text {-Drug release } \\
\text { Franz diffusion } \\
\text { cells } \\
\text { - Mucoadhesion } \\
\text { Texture analyzer } \\
\text {-Antimicrobial } \\
\text { activity } \\
\\
\text { disk diffusion } \\
\text { method }\end{array}$ & - & $\begin{array}{l}\text { - Prolonged drug rel } \\
\text { - Enhanced antimicl } \\
\text { activity against } S \text {. a } \\
\text { and } S \text {. mutans in pr } \\
\text { of chitosan }\end{array}$ \\
\hline
\end{tabular}




\begin{tabular}{|c|c|c|c|c|c|c|}
\hline Drug & Target & Delivery system & Ingredients & In vitro studies & In vivo studies & Results \\
\hline Tetracycline & Periodontal pocket & Nanofiber & $\begin{array}{l}\text { PLGA and gum } \\
\text { tragacanth }\end{array}$ & $\begin{array}{l}\text { - Drug release } \\
\text { (immersing } \\
\text { membrane in } \\
\text { PBS, pH 7.4) } \\
\text { - Biocompatibility } \\
\text { using Human } \\
\text { dermal fibroblast } \\
\text { cells } \\
\text { - The } \\
\text { antibacterial } \\
\text { activity using } \\
\text { agar plate } \\
\text { method }\end{array}$ & - & $\begin{array}{l}\text { - Sustained release } \\
\text { days } \\
\text { - Biocompatible } \\
\text { - Antibacterial activi } \\
\text { against } S \text {. aureus a } \\
\text { aeruginosa }\end{array}$ \\
\hline Tetracycline & Oral mucosa & Nanofiber & Chitosan and PVA & $\begin{array}{l}\text { - Drug release } \\
\text { Vial method } \\
\text { - The } \\
\text { antibacterial } \\
\text { activity (using } \\
\text { samples } \\
\text { collected from } \\
\text { human } \\
\text { periodontal } \\
\text { subgingival } \\
\text { pocket of } \\
\text { patients with } \\
\text { chronic } \\
\text { periodontitis) } \\
\text { - Cytotoxicity } \\
\text { analysis MTT } \\
\text { assay (neonatal } \\
\text { human dermal } \\
\text { fibroblast cells) }\end{array}$ & - & $\begin{array}{l}\text { - Sustained release } \\
\text { days } \\
\text { - Antibacterial activi } \\
\text { against } F \text {. nucleatu } \\
\text { micra, } P \text {. nigrescens } \\
\text { intermedia, E. noda } \\
\text { gracilis, } C \text {. rectus ar } \\
\text { showae, T. denticoli } \\
\text { forsythia and } P \text { gin! } \\
\text { - No cytotoxic effec }\end{array}$ \\
\hline Tetracycline & Implant surface & Nanofiber & PLA, PCL, and gelatin & $\begin{array}{l}\text { - Antimicrobial } \\
\text { activity agar } \\
\text { diffusion assay } \\
\text { - Murine derived } \\
\text { osteoprecursor } \\
\text { cell (MC3T3-E1) } \\
\text { response }\end{array}$ & - & $\begin{array}{l}\text { - Antimicrobial activ } \\
\text { against } A \text {. } \\
\text { actinomycetemcom } \\
F \text {. nucleatum, P. gin! } \\
\text { and } P \text {. intermedia } \\
\text { - Significant increas } \\
\text { alkaline phosphata: } \\
\text { levels indicating an } \\
\text { osteogenic differen }\end{array}$ \\
\hline Antiinflammatc & agents & & & & & \\
\hline $\begin{array}{l}\text { Aspirin and } \\
\text { erythropoietin }\end{array}$ & $\begin{array}{l}\text { Submucoperiosteous } \\
\text { tissue }\end{array}$ & Hydrogel & $\begin{array}{l}\text { Chitosan, } \beta \text {-sodium } \\
\text { glycerophosphate, } \\
\text { gelatin }\end{array}$ & $\begin{array}{l}\text { - Drug release } \\
\text { (adding PBS to } \\
\text { hydrogel } \\
\text { containing } \\
\text { plates) } \\
\text { - Cytotoxicity } \\
\text { MTT assay (rat } \\
\text { bone marrow } \\
\text { stromal cells) }\end{array}$ & $\begin{array}{l}\text { Ligature induced } \\
\text { periodontitis in } \\
\text { nude mice and } \\
\text { Wistar rats }\end{array}$ & $\begin{array}{l}\text { - Sustained release } \\
\text { days } \\
\text { - Anti-inflammatory } \\
\text { activity and signific } \\
\text { periodontium regen } \\
\text { - No cytotoxicity }\end{array}$ \\
\hline Tenoxicam & Buccal mucosa & Film & Chitosan & $\begin{array}{l}\text { - Drug release } \\
\text { study } \\
\text { (immersing films } \\
\text { in artificial } \\
\text { saliva) }\end{array}$ & $\begin{array}{l}\text { Healthy } \\
\text { volunteers } \\
\text { - Mucoadhesion }\end{array}$ & $\begin{array}{l}\text { - Controlled release } \\
\text { - Mucoadhesion tim } \\
1.25 \pm 0.17 \mathrm{~h}\end{array}$ \\
\hline
\end{tabular}




\begin{tabular}{|c|c|c|c|c|c|c|}
\hline Drug & Target & Delivery system & Ingredients & In vitro studies & In vivo studies & Results \\
\hline \multirow[t]{3}{*}{ Atorvastatin } & \multirow[t]{3}{*}{ Periodontal pocket } & \multirow[t]{3}{*}{ Gel } & \multirow[t]{3}{*}{$\begin{array}{l}\text { Base and water soluble } \\
\text { chitosan }\end{array}$} & \multirow[t]{3}{*}{-} & \multirow{3}{*}{$\begin{array}{l}\text { Ligature induced } \\
\text { periodontitis in } \\
\text { Wistar rats } \\
\text { - } \\
\text { Antiinflammatory } \\
\text { and osteoclastic } \\
\text { activity }\end{array}$} & $\begin{array}{l}\text { - Enhanced anti- } \\
\text { inflammatory effec } \\
\text { presence of chitosa }\end{array}$ \\
\hline & & & & & & $\begin{array}{l}\text { - Bone and tissue h } \\
\text { after week } 3\end{array}$ \\
\hline & & & & & & $\begin{array}{l}\text { - No difference betv } \\
\text { water soluble and b } \\
\text { chitosan }\end{array}$ \\
\hline \multirow{6}{*}{$\begin{array}{l}\text { Atorvastatin } \\
\text { and } \\
\text { atorvastatin } \\
\text { solid } \\
\text { dispersions }\end{array}$} & \multirow[t]{6}{*}{ Periodontal pocket } & \multirow[t]{6}{*}{ Gel } & \multirow{6}{*}{$\begin{array}{l}\text { Base and water soluble } \\
\text { chitosan }\end{array}$} & -Drug release & \multirow[t]{6}{*}{-} & - Prolonged drug re \\
\hline & & & & $\begin{array}{l}\text { Franz diffusion } \\
\text { cells }\end{array}$ & & $\begin{array}{l}\text { - Suitable mucoadh } \\
\text { and syringability }\end{array}$ \\
\hline & & & & $\begin{array}{l}\text { - Mucoadhesion } \\
\text { and syringability }\end{array}$ & & $\begin{array}{l}\text { - Decreased release } \\
\text { inflammatory cytok } \\
(\|-1 \beta, L-6,-8) \text { an }\end{array}$ \\
\hline & & & & Texture analyzer & & inflammatory cytok \\
\hline & & & & $\begin{array}{l}\text { - Anti- } \\
\text { inflammatory } \\
\text { activity }\end{array}$ & & $\begin{array}{l}\text { TGF- } \beta 3) \text {, enhanced } \\
\text { presence of chitosa }\end{array}$ \\
\hline & & & & $\begin{array}{l}\text { human gingival } \\
\text { fibroblast } \\
\text { induced cells }\end{array}$ & & $\begin{array}{l}\text { - No difference betv } \\
\text { atorvastatin and so } \\
\text { atorvastatin solid } \\
\text { dispersions }\end{array}$ \\
\hline \multicolumn{7}{|l|}{$\begin{array}{l}\text { Natural } \\
\text { products }\end{array}$} \\
\hline \multirow{8}{*}{$\begin{array}{l}\text { Ziziphus jujuba } \\
\text { extract }\end{array}$} & \multirow[t]{8}{*}{ Buccal mucosa } & \multirow{8}{*}{$\begin{array}{l}\text { Nanofibrous } \\
\text { membrane }\end{array}$} & \multirow{8}{*}{$\begin{array}{l}\text { - Carbopol, } \\
\text { polyacrylonitrile }\end{array}$} & - Drug release & \multirow[t]{8}{*}{-} & \multirow{8}{*}{$\begin{array}{l}\text { - } 80 \% \text { drug release } \\
\text { - Suitable mucoadh } \\
\text { - Improved antimicr } \\
\text { activity against } P \text {. } \\
\text { gingivalis and } F \text {. } \\
\text { nucleatum } \\
\text { - Improved anti- } \\
\text { inflammatory funct } \\
\text { HUVEC }\end{array}$} \\
\hline & & & & (immersing & & \\
\hline & & & & $\begin{array}{l}\text { artificial saliva, } \\
\mathrm{pH} 6.9 \text { ) }\end{array}$ & & \\
\hline & & & & $\begin{array}{l}\text { - Mucoadhesion } \\
\text { using Universal } \\
\text { Testing Machine }\end{array}$ & & \\
\hline & & & & $\begin{array}{l}\text {-Antimicrobial } \\
\text { activity against } \\
\text { using the disk } \\
\text { diffusion } \\
\text { susceptibility test }\end{array}$ & & \\
\hline & & & & $\begin{array}{l}\text { - Anti- } \\
\text { inflammatory } \\
\text { activity permm }\end{array}$ & & \\
\hline & & & & $\begin{array}{l}\text { Permeability } \\
\text { assay }\end{array}$ & & \\
\hline & & & & $\begin{array}{l}\text { (Human } \\
\text { umbilical vein } \\
\text { endothelial cells- } \\
\text { HUVEC) }\end{array}$ & & \\
\hline $\begin{array}{l}\text { Scutellaria } \\
\text { baicalensis and } \\
\text { chlorhexidine }\end{array}$ & Buccal mucosa & Nanoparticle & Water-Ethanol & $\begin{array}{l}\text {-Antibacterial } \\
\text { activity broth } \\
\text { microdilution } \\
\text { assay }\end{array}$ & - & $\begin{array}{l}\text { - Inhibition of biofill } \\
\text { S. mutans, } S \text {. sobrir } \\
\text { nucleatum, and } A \text {. } \\
\text { actinomycetemcon }\end{array}$ \\
\hline $\begin{array}{l}\text { Eucalyptol, } \\
\text { menthol, } \\
\text { thymol }\end{array}$ & Oropharynx & Mouthwash & $\begin{array}{l}\text { Alcohol, benzoic acid, } \\
\text { methyl salicylate, } \\
\text { poloxamer } 407\end{array}$ & $\begin{array}{l}\text { - Antimicrobial } \\
\text { activity agar } \\
\text { plate test }\end{array}$ & $\begin{array}{l}\text { - Male patients } \\
\text { with pharyngeal } \\
\text { gonorrhoea }\end{array}$ & $\begin{array}{l}\text { - Significant reducti } \\
\text { total } N \text {. gonorrhoea } \\
\text { counts in vitro after } \\
\text { exposure }\end{array}$ \\
\hline $\begin{array}{l}\text { fluoride, } \\
\text { eucalyptol, } \\
\text { menthol, } \\
\text { thymol }\end{array}$ & & & & & & $\begin{array}{l}\text { - Significantly reduc } \\
\text { count of } N \text {. gonorrh } \\
\text { on the pharyngeal }\end{array}$ \\
\hline
\end{tabular}




\begin{tabular}{|c|c|c|c|c|c|c|}
\hline Drug & Target & Delivery system & Ingredients & In vitro studies & In vivo studies & Results \\
\hline Propolis & Periodontal pocket & $\begin{array}{l}\text { Magnetic } \\
\text { nanoparticle in } \\
\text { liquid crystalline }\end{array}$ & $\begin{array}{l}\text { Nanoparticle: } \\
\text { Iron oxide } \\
\text { Liquid crystal: } \\
\text { Isopropyl myristate, } \\
\text { polyoxyethylene oleyl } \\
\text { ether }\end{array}$ & $\begin{array}{l}\text { - Drug release } \\
\text { periodontal } \\
\text { pocket simulator } \\
\text { apparatus with a } \\
\text { flow system } \\
\text { - Antifungal } \\
\text { activity broth } \\
\text { macrodilution } \\
\text { test } \\
\text { - Cytotoxicity } \\
\text { fibroblasts cell } \\
\text { line (ATCC CCL- } \\
\text { 1.3) }\end{array}$ & - & $\begin{array}{l}\text { - Prolonged drug rel } \\
\text { - Fungicide activity } \\
\text { Candida spp. } \\
\text { - Very low cytotoxic }\end{array}$ \\
\hline $\begin{array}{l}\text { Green tea } \\
\text { Catechin }\end{array}$ & Periodontal pocket & Strip & $\begin{array}{l}\text { Hydroxypropyl } \\
\text { cellulose }\end{array}$ & - & $\begin{array}{l}\text { - Patients with } \\
\text { advanced } \\
\text { periodontitis } \\
\text { - Antimicrobial } \\
\text { study } \\
\text { Gingival } \\
\text { crevicular fluid } \\
\text { (GCF) } \\
\text { - The pocket } \\
\text { depths (PD) } \\
\text { measured using } \\
\text { a standard } \\
\text { periodontal } \\
\text { probe }\end{array}$ & $\begin{array}{l}\text { - Reduced pocket } \mathrm{d} \epsilon \\
\text { - Decrease in propol } \\
\text { Prevotella spp. and } \\
\text { gingivalis }\end{array}$ \\
\hline Curcumin & Periodontal pocket & Sponge & Collagen & - & $\begin{array}{l}\text { Patients with } \\
\text { chronic } \\
\text { periodontitis } \\
\text { - Plaque index, } \\
\text { gingival index, } \\
\text { probing pocket } \\
\text { depth and } \\
\text { clinical } \\
\text { attachment } \\
\text { levels } \\
\text { - microbiology } \\
\text { N-benzoyl-DL- } \\
\text { arginine- } \beta- \\
\text { naphthylamide } \\
\text { (BANA) test and } \\
\text { microbial colony } \\
\text { count }\end{array}$ & $\begin{array}{l}\text {-Significant reductic } \\
\text { clinical and } \\
\text { microbiological } \\
\text { parameters, yet, low } \\
\text { efficacy when comr } \\
\text { to chlorhexidine chi }\end{array}$ \\
\hline Resveratrol & Periodontal pocket & Nanofiber & Polycaprolactone & $\begin{array}{l}\text { - Drug release } \\
\text { using USP } \\
\text { Apparatus II } \\
\text { - Morphology }\end{array}$ & - & $\begin{array}{l}\text { - Rapid release in th } \\
4 \mathrm{~h} \text {, followed by a } \\
\text { prolonged release u } \\
12 \mathrm{~h}\end{array}$ \\
\hline $\begin{array}{l}\text { Royal Jelly } \\
\text { (bee product) }\end{array}$ & Oral mucosa & Film & $\begin{array}{l}\text { Chitosan and sodium } \\
\text { alginate }\end{array}$ & $\begin{array}{l}\text { - Drug release } \\
\text { modified JP XIV } \\
\text { dissolution } \\
\text { apparatus }\end{array}$ & $\begin{array}{l}\text { 5-fluorouracil } \\
\text { and mild } \\
\text { abrasion induced } \\
\text { oral mucositis in } \\
\text { seven-week-old } \\
\text { Golden Syrian } \\
\text { hamsters } \\
\text { - } \\
\text { Myeloperoxidase } \\
\text { activity (MPO) } \\
\text { - Microscobic } \\
\text { and macroscopic } \\
\text { evaluations } \\
\text { - } \\
\text { Antiinflammatory } \\
\text { activity } \\
\text { Pro-inflammatory } \\
\text { cytokines (TNF-a, } \\
\text { interleukin-1 } \beta \text { ) }\end{array}$ & $\begin{array}{l}\text { - Drug release for } 4 \\
\text { - Decrease in MPO a } \\
\text { - Improved recovery, } \\
\text { day } 8 \\
\text {-Induction of pro- } \\
\text { inflammatory cytok }\end{array}$ \\
\hline
\end{tabular}

*PVA: Polyvinyl alcohol, PLGA: Poly(lactic-co-glycolic acid), PLA: Polylactic acid, PCL: Polycaprolactone, PVP: Polyvinylpyrrolidone, PEG: Polyethylene glycol, Hydroxypropyl methylcellulose, TPP: Tripolyphosphate pentasodium, TNF: Tumor necrosis factor, IL: Interleukin, MTT: Dimethylthiazol-diphenyltetrazolium bI 


\begin{tabular}{|c|c|c|c|c|c|c|}
\hline Drug & Target & Delivery system & Ingredients & In vitro studies & In vivo studies & Results \\
\hline \multicolumn{7}{|l|}{ Miscellaneous } \\
\hline \multirow[t]{5}{*}{ Metformin } & \multirow[t]{5}{*}{ Periodontal pocket } & \multirow[t]{5}{*}{ Film } & \multirow[t]{5}{*}{ Chitosan } & - Drug release & \multirow{5}{*}{$\begin{array}{l}\text { Ligature induced } \\
+ \text { LPS injected } \\
\text { periodontitis in } \\
\text { Wistar rats }\end{array}$} & - Sustained drug rel \\
\hline & & & & Vial method & & \\
\hline & & & & \multirow{3}{*}{$\begin{array}{l}\text { - Antibacterial } \\
\text { activity disc } \\
\text { diffusion method }\end{array}$} & & $\begin{array}{l}\text { - Antibacterial activi } \\
\text { against } P \text {. gingivalis }\end{array}$ \\
\hline & & & & & & forsythia \\
\hline & & & & & & $\begin{array}{l}\text { - Effectively reduce } \\
\text { alveolar bone destrı }\end{array}$ \\
\hline \multirow[t]{2}{*}{$\begin{array}{l}\text { Lactobacillus } \\
\text { fermentum }\end{array}$} & \multirow[t]{2}{*}{ Oral cavity } & \multirow[t]{2}{*}{ Film } & \multirow[t]{2}{*}{ Carboxymethylcellulose } & \multirow{2}{*}{$\begin{array}{l}\text { - Probiotic } \\
\text { bacteria release } \\
\text { study (in } \\
\text { simulated } \\
\text { salivary fluid) }\end{array}$} & \multirow[t]{2}{*}{-} & $\begin{array}{l}\text { - Complete bacteria } \\
\text { release in } 4 \mathrm{~min}\end{array}$ \\
\hline & & & & & & $\begin{array}{l}\text { - Maintenance of pr } \\
\text { viability and antioxi } \\
\text { activity }\end{array}$ \\
\hline \multirow[t]{2}{*}{$\begin{array}{l}\text { Bismuth } \\
\text { subsalicylate }\end{array}$} & \multirow[t]{2}{*}{ Oral mucosa } & \multirow[t]{2}{*}{ Nanoparticle } & & $\begin{array}{l}\text { - Antibacterial } \\
\text { activity agar } \\
\text { diffusion }\end{array}$ & \multirow[t]{2}{*}{-} & $\begin{array}{l}\text { - High antibacterial } \\
\text { against } A \text {. } \\
\text { actinomycetemcom }\end{array}$ \\
\hline & & & & $\begin{array}{l}\text { - Cytotoxicity } \\
\text { using human } \\
\text { gingival } \\
\text { fibroblast (HGF- } \\
\text { 1) cell line }\end{array}$ & & $\begin{array}{l}\text { Gingivalis } \\
\text { - Low cytotoxicity }\end{array}$ \\
\hline \multirow{2}{*}{$\begin{array}{l}\text { PolymP-n } \\
\text { Active } \\
\text { nanoparticles } \\
\text { with silver and } \\
\text { doxycycline }\end{array}$} & \multirow[t]{2}{*}{$\begin{array}{l}\text { Coating } \\
\text { hydroxyapatite discs }\end{array}$} & \multirow[t]{2}{*}{ Nanoparticles } & \multirow[t]{2}{*}{-} & $\begin{array}{l}\text { - Anti-biofilm } \\
\text { activity }\end{array}$ & \multirow[t]{2}{*}{-} & $\begin{array}{l}\text { - Destruction of biof } \\
\text { formation }\end{array}$ \\
\hline & & & & $\begin{array}{l}\text { - Antibacterial } \\
\text { activity agar } \\
\text { diffusion }\end{array}$ & & $\begin{array}{l}\text { - Antibacterial activi } \\
\text { against } S \text {. oralis, } A \text {. } \\
\text { naeslundii, V. parvu } \\
\text { nucleatum, P. gingil } \\
\text { and } A \text {. } \\
\text { actinomycetemcom }\end{array}$ \\
\hline \multirow[t]{2}{*}{$\mathrm{Fe}_{3} \mathrm{O}_{4}$} & \multirow[t]{2}{*}{ Dentinal tubule } & \multirow[t]{2}{*}{ Liposome } & \multirow[t]{2}{*}{ PEG } & $\begin{array}{l}\text { - Ex-vivo } \\
\text { evaluation in } \\
\text { extracted }\end{array}$ & \multirow[t]{2}{*}{-} & $\begin{array}{l}\text { - Diffusion into dent } \\
\text { tubules }\end{array}$ \\
\hline & & & & human teeth & & \\
\hline \multirow[t]{2}{*}{$\begin{array}{l}\text { Indocyanine } \\
\text { green }\end{array}$} & \multirow[t]{2}{*}{ Oral cavity } & \multirow[t]{2}{*}{ Nanosphere } & \multirow[t]{2}{*}{ PLGA, chitosan } & $\begin{array}{l}\text { - Antibacterial } \\
\text { activity activity }\end{array}$ & - & $\begin{array}{l}\text { - Antimicrobial effer } \\
\text { gingivalis with }\end{array}$ \\
\hline & & & & blood agar plates & & $\begin{array}{l}\text { Nano/c with low-le } \\
\text { diode laser }(0.5 \mathrm{~W} ; \\
805 \mathrm{~nm} \text { ) irradiation }\end{array}$ \\
\hline \multirow{7}{*}{$\begin{array}{l}\text { Pac-525 } \\
\text { (Antimicrobial } \\
\text { peptide) }\end{array}$} & \multirow[t]{7}{*}{ Oral mucosa } & \multirow[t]{7}{*}{ Nanofiber } & $\begin{array}{l}\text { Composite membrane: } \\
\text { Gelatin/Chitosan }\end{array}$ & $\begin{array}{l}\text { - Drug release } \\
\text { studv (immersing }\end{array}$ & - & - A rapid release in t \\
\hline & & & $\begin{array}{l}\text { Hydroxyapatite } \\
\text { nanoparticles }\end{array}$ & $\begin{array}{l}\text { membrane in } \\
\text { PBS, pH 7.4) }\end{array}$ & & $\begin{array}{l}\text { release at around } 4 \\
\text { followed by a long-t } \\
\text { sustained release }\end{array}$ \\
\hline & & & Microspheres: PLGA & $\begin{array}{l}\text { - Osteogenic } \\
\text { activity using rat }\end{array}$ & & $\begin{array}{l}\text { - Promoted osteoge } \\
\text { differentiation }\end{array}$ \\
\hline & & & & $\begin{array}{l}\text { bone marrow } \\
\text { mesenchymal } \\
\text { stem cells } \\
\text { (rBMSCs) }\end{array}$ & & \\
\hline & & & & $\begin{array}{l}\text { - The } \\
\text { antibacterial } \\
\text { activity using } \\
\text { agar diffusion } \\
\text { method }\end{array}$ & & $\begin{array}{l}\text { - A good antibacteri } \\
\text { activity against } S \text {. a }\end{array}$ \\
\hline & & & & & & and \\
\hline & & & & & & E. coli up to one mo \\
\hline
\end{tabular}

*PVA: Polyvinyl alcohol, PLGA: Poly(lactic-co-glycolic acid), PLA: Polylactic acid, PCL: Polycaprolactone, PVP: Polyvinylpyrrolidone, PEG: Polyethylene glycol, Hydroxypropyl methylcellulose, TPP: Tripolyphosphate pentasodium, TNF: Tumor necrosis factor, IL: Interleukin, MTT: Dimethylthiazol-diphenyltetrazolium bI 
Table 2

Drug delivery systems for treatment of viral infection-related conditions in the oral cavity*

\begin{tabular}{|c|c|c|c|c|c|c|c|c|}
\hline Virus & Drug & Target & $\begin{array}{l}\text { Delivery } \\
\text { system }\end{array}$ & Ingredients & In vitro studies & In vivo studies & Results & Reference \\
\hline HSV & Acyclovir & $\begin{array}{l}\text { Oral } \\
\text { mucosa }\end{array}$ & In situ gel & $\begin{array}{l}\text { Poloxamer 407, Carbopol } \\
\text { 934, and HPMC }\end{array}$ & $\begin{array}{l}\text {-Drug release } \\
\text {-Ex-vitro } \\
\text { (porcine oral } \\
\text { mucosa) drug } \\
\text { permeation } \\
\text { - } \\
\text { Mucoadhesion } \\
\text { (porcine oral } \\
\text { mucosa) } \\
\text { using modified } \\
\text { physical } \\
\text { balance }\end{array}$ & - & $\begin{array}{l}\text {-Drug release up } \\
\text { to } 6 \mathrm{~h} \\
\text {-Suitable } \\
\text { mucoadhesion }\end{array}$ & [204] \\
\hline $\begin{array}{l}\text { HSV- } \\
1 / 2\end{array}$ & Acyclovir & $\begin{array}{l}\text { Buccal } \\
\text { mucosa }\end{array}$ & $\begin{array}{l}\text { Films } \\
\text { impregnated } \\
\text { with } \\
\text { nanospheres }\end{array}$ & $\begin{array}{l}\text { Nanosphere: PLGA, PVA } \\
\text { Film: } \\
\text { HPMC K15, Eudragit RL } \\
\text { 100, Carbopol 974P, PEG } \\
\text { 200, Ethyl cellulose }\end{array}$ & $\begin{array}{l}\text {-Drug release } \\
\text { paddle over } \\
\text { disc method } \\
\text { using USP II } \\
\text { apparatus } \\
\text {-Drug } \\
\text { permeation } \\
\text { Franz } \\
\text { diffusion cells } \\
\text { - } \\
\text { Mucoadhesion } \\
\text { Texture } \\
\text { analyzer using } \\
\text { rabbit buccal } \\
\text { mucosa }\end{array}$ & $\begin{array}{l}\text { White male } \\
\text { rabbits }\end{array}$ & $\begin{array}{l}\text { - High permeation } \\
\text { and controlled } \\
\text { release of drug } \\
\text { over an extended } \\
\text { period of time } \\
\text { - Enhanced } \\
\text { bioavailability by } \\
\sim 8 \text { fold }\end{array}$ & [205] \\
\hline HSV-1 & Acyclovir & $\begin{array}{l}\text { Buccal } \\
\text { mucosa }\end{array}$ & $\begin{array}{l}\text { Tablet } \\
\text { (Sitavig®) }\end{array}$ & $\begin{array}{l}\text { Hypromellose, milk } \\
\text { protein concentrate, } \\
\text { sodium lauryl sulfate, } \\
\text { magnesium stearate, } \\
\text { MCC, povidone, colloidal } \\
\text { silicon dioxide }\end{array}$ & - & $\begin{array}{l}\text { Patients, with at } \\
\text { least four } \\
\text { herpes episodes } \\
\text { in the previous } \\
\text { year }\end{array}$ & $\begin{array}{l}\text { - Prolonged } \\
\text { plasma drug } \\
\text { levels } \\
\text { - Reduction of } \\
\text { duration of the } \\
\text { herpes } \\
\text { episode } \\
\text { - Less primary } \\
\text { vesicular lesions }\end{array}$ & [206] \\
\hline HSV-1 & $\begin{array}{l}\text { Penciclovir } \\
\text { Acyclovir }\end{array}$ & - & Cream & - & - & $\begin{array}{l}\text { Patients with } \\
\text { herpes simplex } \\
\text { facialis/labialis } \\
\text { (five times daily } \\
\text { for } 7 \text { days) }\end{array}$ & $\begin{array}{l}-1 \% \text { penciclovir } \\
\text { and } 3 \% \text { acyclovir } \\
\text { equally effective }\end{array}$ & [207] \\
\hline $\begin{array}{l}\text { HHV-4 } \\
\text { (EBV) }\end{array}$ & $\begin{array}{l}\text { Podophyllin } \\
\text { resin } \\
\text { Penciclovir } \\
\text { Acyclovir }\end{array}$ & Tongue & Cream & - & - & $\begin{array}{l}\text { Patients with } \\
\text { HIV infection } \\
\text { and oral hairy } \\
\text { leukoplakia } \\
\text { (related to EBV) }\end{array}$ & $\begin{array}{l}\text { - Effective clinical } \\
\text { healing within 7- } \\
8 \text { weeks } \\
\text { - Faster clinical } \\
\text { healing with } \\
\text { podophyllin resin } \\
\text { + acyclovir }\end{array}$ & [208] \\
\hline $\begin{array}{l}\text { HHV-5 } \\
(\mathrm{CMV})\end{array}$ & $\begin{array}{l}\text { Plasmid DNA } \\
\text { (CMV- } \beta \text {-gal) } \\
\text { and } \beta- \\
\text { galactosidase }\end{array}$ & $\begin{array}{l}\text { Buccal } \\
\text { mucosa }\end{array}$ & Bilayer film & $\begin{array}{l}\text { Polycarbophil and } \\
\text { Eudragit S-100 }\end{array}$ & $\begin{array}{l}\text { - Drug release } \\
\text { vial method } \\
\text { - } \\
\text { Mucoadhesion } \\
\text { time using a } \\
\text { glass model }\end{array}$ & $\begin{array}{l}\text { Female New } \\
\text { Zealand White } \\
\text { rabbits }\end{array}$ & $\begin{array}{l}\text { - IgG titers } \\
\text { comparable to } \\
\text { that of } \\
\text { subcutaneous } \\
\text { administration }\end{array}$ & [209] \\
\hline
\end{tabular}


Table 3

Drug delivery systems for treatment of fungal infection-related conditions in the oral cavity*

\begin{tabular}{|c|c|c|c|c|c|c|c|c|}
\hline Fungus & Drug & Target & $\begin{array}{l}\text { Delivery } \\
\text { system }\end{array}$ & Ingredients & In vitro studies & In vivo studies & Results & $\mathbf{R}_{\mathbf{r}}$ \\
\hline $\begin{array}{l}\text { Candida } \\
\text { albicans }\end{array}$ & $\begin{array}{l}\text { Chlorhexidine } \\
\text { digluconate }\end{array}$ & Oral cavity & Gel, film & $\begin{array}{l}\text { Chitosan, glycerin, TPP, } \\
\text { lactic acid }\end{array}$ & $\begin{array}{l}\text { - Drug release } \\
\text { Franz } \\
\text { diffusion cells } \\
\text { - Antifungal } \\
\text { activity }\end{array}$ & - & $\begin{array}{l}\text { - Prolonged } \\
\text { drug release } \\
\text { - Enhanced } \\
\text { antifungal } \\
\text { activity } \\
\text { obtained in } \\
\text { presence of } \\
\text { chitosan }\end{array}$ & \\
\hline $\begin{array}{l}\text { Candida } \\
\text { albicans }\end{array}$ & Nystatin & $\begin{array}{l}\text { Buccal } \\
\text { mucosa }\end{array}$ & Gel, film & $\begin{array}{l}\text { Chitosan, glycerin, TPP, } \\
\text { lactic acid, glacial } \\
\text { acetic acid, aspartame }\end{array}$ & $\begin{array}{l}\text { - Drug release } \\
\text { Franz } \\
\text { diffusion cells }\end{array}$ & $\begin{array}{l}\text { - Young male } \\
\text { golden Syrian } \\
\text { hamsters } \\
\text { (5-fluorouracil } \\
\text { induced } \\
\text { mucositis) } \\
\text { - Healthy } \\
\text { volunteers }\end{array}$ & $\begin{array}{l}\text { - Prolonged } \\
\text { release } \\
\text { - Increased } \\
\text { reduction of } \\
\text { granulation } \\
\text { tissue and } \\
\text { formation of } \\
\text { scar tissue in } \\
\text { presence of } \\
\text { chitosan } \\
\text { - Drug } \\
\text { concentrations } \\
\text { above MIC } \\
\text { value for } \\
\text { maintained for } \\
90 \text { min at the } \\
\text { application } \\
\text { site }\end{array}$ & [9 \\
\hline $\begin{array}{l}\text { Candida } \\
\text { albicans }\end{array}$ & $\begin{array}{l}\text { Ciclopirox } \\
\text { olamine }\end{array}$ & $\begin{array}{l}\text { Buccal } \\
\text { mucosa }\end{array}$ & Bilayer film & $\begin{array}{l}\text { Polyethylene oxide, } \\
\text { Eudragit, glycerol }\end{array}$ & $\begin{array}{l}\text { - Drug release } \\
\text { USP paddle } \\
\text { apparatus } \\
\text { - Drug } \\
\text { permeation } \\
\text { (porcine } \\
\text { buccal } \\
\text { mucosa) } \\
\text { using modified } \\
\text { Franz cells }\end{array}$ & $\begin{array}{l}\text { White SPF } \\
\text { European } \\
\text { rabbits } \\
\text { intraorally } \\
\text { infected with } \\
\text { Candida } \\
\text { albicans } \\
\text { (ATCC90028) }\end{array}$ & $\begin{array}{l}\text { - Drug release } \\
\text { for } 12 \mathrm{~h} \\
\text { - } \\
\text { Accumulation } \\
\text { of drug in } \\
\text { porcine buccal } \\
\text { mucosa in ex } \\
\text { vivo studies. } \\
\text { - Prolonged } \\
\text { plasma levels } \\
\text { - Progressive } \\
\text { healing in } \\
\text { stomatitis } \\
\text { without organ } \\
\text { pathologies }\end{array}$ & {$[2$} \\
\hline $\begin{array}{l}\text { Candida } \\
\text { albicans }\end{array}$ & Clotrimazole & $\begin{array}{l}\text { Buccal } \\
\text { mucosa }\end{array}$ & $\begin{array}{l}\text { pH triggered in- } \\
\text { situ gel } \\
\text { lon triggered in- } \\
\text { situ gel }\end{array}$ & $\begin{array}{l}\text { Carbopol - HPMC } \\
\text { Gellan gum-HPMC }\end{array}$ & $\begin{array}{l}\text { - Drug release } \\
\text { using flow } \\
\text { through device } \\
\text { cell } \\
\text { - Antifungal } \\
\text { activity } \\
\text { agar diffusion } \\
\text { method }\end{array}$ & - & $\begin{array}{l}\text { - Prolonged } \\
\text { release }(6 \mathrm{~h}) \text { in } \\
\text { presence of } \\
\text { gellan gum } \\
\text { - Comparable } \\
\text { antifungal } \\
\text { activity to that } \\
\text { of a } \\
\text { commercial } \\
\text { product }\end{array}$ & [2 \\
\hline
\end{tabular}

*PLA: Polylactic acid; PLGA: Polylactic-co-glycolic acid; TPP: Tripolyphosphate pentasodium; HPMC: Hydroxypropyl methylcellulose; MIC: Minimum inhibitor] concentration; MCC: Microcrystalline cellulose; PEG: Polyethylene glycol; MTT: Dimethylthiazol-diphenyltetrazolium bromide 


\begin{tabular}{|c|c|c|c|c|c|c|c|c|}
\hline Fungus & Drug & Target & $\begin{array}{l}\text { Delivery } \\
\text { system }\end{array}$ & Ingredients & In vitro studies & In vivo studies & Results & $\mathbf{R}_{\mathbf{l}}$ \\
\hline $\begin{array}{l}\text { Candida } \\
\text { albicans }\end{array}$ & Fluconazole & $\begin{array}{l}\text { Buccal } \\
\text { mucosa }\end{array}$ & Oral strip & $\begin{array}{l}\text { Eudragit RS 100, } \\
\text { Eudragit RL 100, HPMC } \\
\text { E50, HPMC K100M, } \\
\text { PEG } 400\end{array}$ & $\begin{array}{l}\text { - Drug release } \\
\text { dialysis bag } \\
\text { - Ex vivo drug } \\
\text { permeation } \\
\text { (bovine buccal } \\
\text { mucosa) } \\
\text { using Franz } \\
\text { diffusion cells } \\
\text { - Antifungal } \\
\text { activity (agar } \\
\text { well diffusion } \\
\text { method) } \\
\text { - } \\
\text { Mucoadhesion }\end{array}$ & - & $\begin{array}{l}\text { - Fast } \\
\text { disintegration } \\
(5-30 \mathrm{~s}), \\
\text { prolonged } \\
\text { release } \\
\text { - Enhanced } \\
\text { antifungal } \\
\text { activity } \\
\text { - Suitable } \\
\text { mucoadhesion } \\
\text { with Eudragit } \\
\text { and HPMC } \\
\text { combination } \\
\text {-No drug } \\
\text { permeation } \\
\text { across bovine } \\
\text { buccal } \\
\text { mucosa }\end{array}$ & {$[2$} \\
\hline & & & & & $\begin{array}{l}\text { texture } \\
\text { analyzer } \\
\text { - Cytotoxicity } \\
\text { MTT assay } \\
\text { with Chinese } \\
\text { hamster ovary } \\
\text { (CHO) cells }\end{array}$ & & $\begin{array}{l}\text { - No cytotoxic } \\
\text { effect }\end{array}$ & \\
\hline $\begin{array}{l}\text { Candida } \\
\text { albicans }\end{array}$ & Miconazole & $\begin{array}{l}\text { Sublingual } \\
\text { and buccal } \\
\text { mucosa }\end{array}$ & $\begin{array}{l}\text { Nanostructured } \\
\text { lipid carrier } \\
\text { (NLC) based } \\
\text { hydrogel }\end{array}$ & $\begin{array}{l}\text { - Hydrogel: Carbopol } \\
2001(\text { PFC } ₫ \text { ) and } \\
\text { triethanolamine } \\
\text { - NLC: Gelucire }{ }^{\circledR} 43 / 01 \text {, } \\
\text { Miglyol } \AA 812 \text {, Tween }{ }^{\circledR} \\
80\end{array}$ & $\begin{array}{l}\text { - Drug release } \\
\text { dialysis bag } \\
\text { - Antifungal } \\
\text { activity (agar- } \\
\text { well diffusion } \\
\text { method) }\end{array}$ & - & $\begin{array}{l}\text { - Controlled } \\
\text { drug release } \\
(16 \% \text { and } 22 \% \\
\text { in } 48 \mathrm{~h}) \\
\text { - Antifungal } \\
\text { activity with } \\
\text { lowered dose }\end{array}$ & {$[2$} \\
\hline $\begin{array}{l}\text { Candida } \\
\text { albicans }\end{array}$ & $\begin{array}{l}\text { Miconazole } \\
\text { Clotrimazole }\end{array}$ & $\begin{array}{l}\text { Buccal } \\
\text { mucosa } \\
\text { Oral mucosa }\end{array}$ & $\begin{array}{l}\text { Tablet } \\
\text { Troche }\end{array}$ & $\begin{array}{l}\text { - Croscarmellose } \\
\text { sodium, magnesium } \\
\text { stearate, MCC, } \\
\text { povidone, dextrates }\end{array}$ & - & $\begin{array}{l}\text { - HIV positive } \\
\text { patients with } \\
\text { oropharyngeal } \\
\text { candidiasis ( } \geq \\
18 \text { years of } \\
\text { age) } \\
\text { - Buccal tablet } \\
\text { adhesion time } \\
\text { - Local } \\
\text { inflammation } \\
\text { (gingival } \\
\text { index) } \\
\text { - Once daily } \\
\text { buccal tablets } \\
\text { of miconazole } \\
\text { and } 5 \text { times } \\
\text { daily } \\
\text { clotrimazole } \\
\text { troches for } 14 \\
\text { days }\end{array}$ & $\begin{array}{l}\text { - Effective, } \\
\text { safe, and well- } \\
\text { tolerated } \\
\text { treatment with } \\
\text { once-daily } \\
\text { dose buccal } \\
\text { miconazole } \\
\text { - Similar } \\
\text { efficacy } \\
\text { between once- } \\
\text { daily buccal } \\
\text { tablet and } 5 \\
\text { times daily } \\
\text { troche }\end{array}$ & {$[2$} \\
\hline
\end{tabular}




\begin{tabular}{|c|c|c|c|c|c|c|c|c|}
\hline Fungus & Drug & Target & $\begin{array}{l}\text { Delivery } \\
\text { system }\end{array}$ & Ingredients & In vitro studies & In vivo studies & Results & $\mathbf{R}$ \\
\hline $\begin{array}{l}\text { Candida } \\
\text { albicans }\end{array}$ & Natamycin & $\begin{array}{l}\text { Buccal } \\
\text { mucosa }\end{array}$ & Bilayered tablet & Carbopol 974, HPMC & $\begin{array}{l}\text { - Drug release } \\
\text { studies USP } \\
\text { rotating } \\
\text { paddle } \\
\text { method } \\
\text { - Adhesion } \\
\text { (membrane) } \\
\text { using Texture } \\
\text { Analyzer } \\
\text { - Antifungal } \\
\text { activity (broth } \\
\text { microdilution } \\
\text { method) }\end{array}$ & $\begin{array}{l}\text { - Ten females } \\
\text { and two males } \\
(22-29 \text { year- } \\
\text { old) with no } \\
\text { history of dry } \\
\text { mouth } \\
\text { conditions and } \\
\text { oral lesions } \\
\text { - Tablets } \\
\text { placed on } \\
\text { buccal mucosa } \\
\text { - Saliva } \\
\text { samples } \\
\text { collected from } \\
\text { different } \\
\text { regions in the } \\
\text { oral cavity }\end{array}$ & $\begin{array}{l}\text { - Unidirectional } \\
\text { drug release } \\
\text { obtained in } \\
\text { prolonged } \\
\text { fashion } \\
\text { - Drug } \\
\text { concentration } \\
\text { maintained } \\
\text { above the MIC } \\
\text { value } \\
\text {-Highest drug } \\
\text { levels on } \\
\text { application } \\
\text { side, lowest } \\
\text { drug levels in } \\
\text { sublingual } \\
\text { region }\end{array}$ & {$[2$} \\
\hline $\begin{array}{l}\text { Candida } \\
\text { albicans }\end{array}$ & Nystatin & $\begin{array}{l}\text { Buccal } \\
\text { mucosa }\end{array}$ & $\begin{array}{l}\text { Nanoparticles } \\
\text { incorporated in } \\
\text { toothpaste, } \\
\text { oral gel and } \\
\text { oral films }\end{array}$ & $\begin{array}{l}\text { Nanoparticles: } \\
\text { PLA, PLGA } \\
\text { and alginate } \\
\text { Toothpaste: } \\
\text { xanthan gum, } \\
\text { glycerol, } \\
\text { sorbitol, } \\
\text { citric acid } \\
\text { buffer, NaF, } \\
\text { CaCO } 3 \text {, } \\
\text { microcrystalline } \\
\text { cellulose, } \\
\text { sodium } \\
\text { lauryl sulfate } \\
\text { Gel: } \\
\text { Sodium } \\
\text { hydroxide, } \\
\text { Carbopol } 940 \\
\text { Film: HPMC, } \\
\text { glycerol }\end{array}$ & $\begin{array}{l}\text { - } \\
\text { Mucoadhesion } \\
\text { Texture } \\
\text { Analyzer and } \\
\text { retention } \\
\text { studies with } \\
\text { mucus- } \\
\text { secreting } \\
\text { HT29-MTX } \\
\text { cells }\end{array}$ & - & $\begin{array}{l}\text { - Enhanced } \\
\text { mucoadhesion } \\
\text { in order of: } \\
\text { film with the } \\
\text { PLGA } \\
\text { nanoparticles } \\
\text { > gel with PLA } \\
\text { nanoparticles } \\
\text { > toothpaste } \\
\text { with alginate } \\
\text { nanoparticles }\end{array}$ & {$[2$} \\
\hline $\begin{array}{l}\text { Candida } \\
\text { albicans }\end{array}$ & Nystatin & $\begin{array}{l}\text { Buccal } \\
\text { mucosa }\end{array}$ & Microspheres & $\begin{array}{l}\text { Alginate,chitosan, } \\
\text { calcium carbonate, } \\
\text { calcium chloride, acetic } \\
\text { acid, soya oil, and } \\
\text { Span® } 80\end{array}$ & $\begin{array}{l}\text { - Drug release } \\
\text { Franz } \\
\text { diffusion cell } \\
\text { - Antifungal } \\
\text { activity } \\
\text { (Sabouraud } \\
\text { Dextrose } \\
\text { medium) }\end{array}$ & $\begin{array}{l}\text { Female } \\
\text { crossbred } \\
\text { (Landrace x } \\
\text { Large White) } \\
\text { pigs } \\
\text { - Drug plasma } \\
\text { levels } \\
\text { - } \\
\text { Histopathology } \\
\text { after } \\
\text { sacrification }\end{array}$ & $\begin{array}{l}\text { - High fungal } \\
\text { activity } \\
\text { - No nystatin } \\
\text { in systemic } \\
\text { circulation, } \\
\text { assuring the } \\
\text { safety of the } \\
\text { treatment } \\
\text { - Nystatin } \\
\text { retained in the } \\
\text { tissue without } \\
\text { any tissue } \\
\text { damage }\end{array}$ & {$[2$} \\
\hline
\end{tabular}




\begin{tabular}{|c|c|c|c|c|c|c|c|c|}
\hline Fungus & Drug & Target & $\begin{array}{l}\text { Delivery } \\
\text { system }\end{array}$ & Ingredients & In vitro studies & In vivo studies & Results & $\mathbf{R}$ \\
\hline \multirow{3}{*}{$\begin{array}{l}\text { Candida } \\
\text { albicans, } \\
\text { Candida } \\
\text { glabrata, } \\
\text { Candida } \\
\text { parapsilosis }\end{array}$} & \multirow[t]{3}{*}{ Clotrimazole } & \multirow[t]{3}{*}{$\begin{array}{l}\text { Buccal and } \\
\text { sublingual } \\
\text { mucosa }\end{array}$} & \multirow[t]{3}{*}{ Nanoemulsion } & \multirow{3}{*}{$\begin{array}{l}\text { Capry-locaproyl } \\
\text { macrogol-8 glycerides, } \\
\text { medium-chain } \\
\text { triglycerides, propylene } \\
\text { glycol monocaprylate, } \\
\text { propylenglycol }\end{array}$} & $\begin{array}{l}\text { - Drug release } \\
\text { and } \\
\text { permeation }\end{array}$ & \multirow[t]{3}{*}{ - } & $\begin{array}{l}\text { - Prolonged } \\
\text { drug release } \\
(48 \mathrm{~h})\end{array}$ & \multirow[t]{3}{*}{2} \\
\hline & & & & & $\begin{array}{l}\text { Franz } \\
\text { difussion cells } \\
\text { (porcine } \\
\text { buccal and } \\
\text { sublingual } \\
\text { mucosa) }\end{array}$ & & $\begin{array}{l}\text { - Drug } \\
\text { permeation } \\
\text { similar to that } \\
\text { of a } \\
\text { commercial } \\
\text { product }\end{array}$ & \\
\hline & & & & & $\begin{array}{l}\text { - Antifungal } \\
\text { activity (broth } \\
\text { microdilution } \\
\text { method) }\end{array}$ & & $\begin{array}{l}\text { - Significant } \\
\text { antifungal } \\
\text { activity } \\
\text { against } \\
\text { Candida ssp. }\end{array}$ & \\
\hline \multirow[t]{2}{*}{$\begin{array}{l}\text { Candida } \\
\text { albicans, } \\
\text { Candida } \\
\text { parapsilosis, } \\
\text { Candida. } \\
\text { krusei }\end{array}$} & \multirow[t]{2}{*}{ Posaconazole } & \multirow[t]{2}{*}{$\begin{array}{l}\text { Buccal } \\
\text { mucosa }\end{array}$} & \multirow[t]{2}{*}{ Film } & \multirow[t]{2}{*}{$\begin{array}{l}\text { Alginate } \\
\text { oligosaccharides, } \\
\text { sodium alginate, } \\
\text { glycerol }\end{array}$} & $\begin{array}{l}\text { - } \\
\text { uucoadhesion } \\
\text { using (bovine } \\
\text { buccal } \\
\text { mucosa) }\end{array}$ & \multirow[t]{2}{*}{$\begin{array}{l}\text { - Human } \\
\text { volunteers } \\
\text { Mucoadhesion } \\
\text { of placebo } \\
\text { films }\end{array}$} & $\begin{array}{l}\text { - Prolonged } \\
\text { release }(5 \mathrm{~h}) \\
\text { and suitable } \\
\text { mucoadhesive } \\
\text { property }\end{array}$ & \multirow[t]{2}{*}{2} \\
\hline & & & & & $\begin{array}{l}\text { - Antifungal } \\
\text { activity (broth } \\
\text { microdilution } \\
\text { method) }\end{array}$ & & $\begin{array}{l}\text { - Improved } \\
\text { antifungal } \\
\text { activity } \\
\text { against }\end{array}$ & \\
\hline \multirow{2}{*}{$\begin{array}{l}\text { Candida } \\
\text { albicans, } \\
\text { Candida } \\
\text { parapsilosis, } \\
\text { Candida } \\
\text { krusei }\end{array}$} & \multirow[t]{2}{*}{$\begin{array}{l}\text { Amphotericin } \\
\text { B }\end{array}$} & \multirow[t]{2}{*}{$\begin{array}{l}\text { Oropharyngeal } \\
\text { cavity }\end{array}$} & \multirow[t]{2}{*}{ Film } & \multirow{2}{*}{$\begin{array}{l}\text { HPMC acetate } \\
\text { succinate, maltodextrin, } \\
\text { sorbitol, dextran, } \\
\text { microcrystalline } \\
\text { cellulose, sodium } \\
\text { carboxymethylcellulose, } \\
\text { HPC }\end{array}$} & $\begin{array}{l}\text { - } \\
\text { Disintegration } \\
\text { test }\end{array}$ & \multirow[t]{2}{*}{-} & $\begin{array}{l}\text { - Fast } \\
\text { disintegration } \\
(60 \mathrm{~s})\end{array}$ & \multirow[t]{2}{*}{2} \\
\hline & & & & & $\begin{array}{l}\text { - Antifungal } \\
\text { activity (agar } \\
\text { diffusion } \\
\text { assay) }\end{array}$ & & $\begin{array}{l}\text { - High } \\
\text { antifungal } \\
\text { activity }\end{array}$ & \\
\hline \multirow{5}{*}{$\begin{array}{l}\text { Cryptococcus } \\
\text { neoformans, } \\
\text { Candida } \\
\text { albicans, } \\
\text { Sporothrix } \\
\text { schenckii }\end{array}$} & \multirow[t]{5}{*}{$\begin{array}{l}\text { Miconazole } \\
\text { nitrate }\end{array}$} & \multirow[t]{5}{*}{$\begin{array}{l}\text { Buccal } \\
\text { mucosa }\end{array}$} & \multirow[t]{5}{*}{ Gel } & \multirow{5}{*}{$\begin{array}{l}\text { - HPMC, carbopol 940, } \\
\text { methyl paraben, propyl } \\
\text { paraben, PEG 400, } \\
\text { propylene glycol, } \\
\text { hydroxyethyl cellulose, } \\
\text { NaCMC, Tween 20, } \\
\text { Tween 80, } \\
\text { triethanolamine }\end{array}$} & $\begin{array}{l}\text { - Ex vivo } \\
\text { permeation } \\
\text { study (goat }\end{array}$ & \multirow[t]{5}{*}{-} & $\begin{array}{l}\text { - Efficient } \\
\text { permeation }\end{array}$ & \multirow[t]{5}{*}{2} \\
\hline & & & & & $\begin{array}{l}\text { buccal } \\
\text { mucosa) } \\
\text { using modified } \\
\text { USP II type }\end{array}$ & & $\begin{array}{l}\text { - High } \\
\text { adhesion and } \\
\text { strength }\end{array}$ & \\
\hline & & & & & $\begin{array}{l}\text { dissolution } \\
\text { apparatus }\end{array}$ & & $\begin{array}{l}\text { - Broader zone } \\
\text { of growth } \\
\text { inhibition }\end{array}$ & \\
\hline & & & & & $\begin{array}{l}\text { - Strength and } \\
\text { mucoadhesion } \\
\text { studies using } \\
\text { Texture } \\
\text { Analyzer }\end{array}$ & & $\begin{array}{l}\text { compared to } \\
\text { marketed } \\
\text { formulation }\end{array}$ & \\
\hline & & & & & $\begin{array}{l}\text { - Antifungal } \\
\text { activity (agar } \\
\text { diffusion } \\
\text { method) }\end{array}$ & & & \\
\hline
\end{tabular}

*PLA: Polylactic acid; PLGA: Polylactic-co-glycolic acid; TPP: Tripolyphosphate pentasodium; HPMC: Hydroxypropyl methylcellulose; MIC: Minimum inhibitor concentration; MCC: Microcrystalline cellulose; PEG: Polyethylene glycol; MTT: Dimethylthiazol-diphenyltetrazolium bromide

Topical drug delivery systems are traditionally formulated as solid dosage forms (e.g., tablets, wafers, films, fibres and patches), liquid dosage forms (e.g., sprays and drops), and semi-solid dosage forms (e.g., gels, oinments) [41-45]. Conventional topical dosage forms are commonly affected by physiological factors, which can reduce the contact of the formulation with the mucosa and lead to reduced efficacy. Hence, numerous strategies have been proposed in order to overcome these difficulties and improve the retention and permeation of drugs in the oral cavity [46, 32 ]. In $50 \mathrm{~s}$, antimicrobials were incorporated into dental cements and resins in order to provide local drug release of antimicrobials [47]. Recognition of local antimicrobial delivery systems in the management of bacterial infections in the oral cavity resulted in a shift in treatment modalities of dental diseases [48]. Chlorhexidine chip [49], metronidazole oral gel [50] and minocycline dental gel [51] are amongst the first formulations brought to the market.

For a successful local drug delivery, mucoadhesive delivery systems have been widely utilized to avoid the rapid removal of the system from the side of application due to physiological conditions in the oral cavity. Interaction between mucin and mucoadhesive polymer enables the system to remain attached on the application site and also provides prolonged release of drug. Penetration enhancers are also incorporated into delivery systems to generate improved efficacy for both local and systemic drug delivery. In designing a local delivery system, it is also important to take into consideration the condition of the disease, as each condition may require distinct penetration and drug retention/distribution profiles for an optimized efficacy. In most conditions, the drug is required to penetrate to deeper layers of the epithelium. Consequently, regarding all the requirements mentioned above, various delivery systems (e.g. liposomes, polymeric nanoparticles, lipid nanoparticles, hydrogels, fibers, films etc) other than conventional formulations have been investigated for an improved treatment of the oral infections [52]. 


\section{Liposomes}

In dentistry, liposomes have been used topically to control the oral biofilm (preventing caries and gingivitis), to treat oral lesions and periodontitis, and in photodynamic therapy. Liposomes are synthetic nano-sized vesicles consisting of one or more phospholipid bilayers, able to accommodate hydrophilic and lipophilic molecules. Liposomes may be formulated with a range of characteristics including different size, charge and drug retention, which can be customized for a given drug and target site $[53,54]$. In early $80 \mathrm{~s}$, Mezei and Gulasekharam [55, 56] have shown the applicability of liposomes as drug carriers for the topical administration using triamcinolone as a model drug. Later, the potential of liposomes as drug carriers to the ulcerated oral mucosa was investigated in vivo in hamsters using radioactive triamcinolone acetonide palmitate [57]. Liposomes were shown to increase local and decrease systemic drug concentration. In addition, the authors suggested that liposomes decrease drug diffusion into neighboring tissues and localize the drug in the area of inflammation. Proteoliposomes with surface-bound succinylated concanavalin A were prepared to deliver triclosan for elimination of Streptococcus sanguis biofilms [58]. It was shown that triclosan delivered in liposome was a more effective growth inhibitor than free triclosan. Further, reactive liposomes were prepared encapsulating the enzymes, glucose oxidase (GO) and GO in combination with horse radish peroxidase (HRP) to eliminate the biofilms of the oral bacterium Streptococcus gordonii [59]. Increased bacterial inhibition was observed with the reactive liposomes. Antibacterial activity in the presence of saliva was also observed with the reactive liposomes.

Immunoliposomes were developed to increase the specificity and affinity of bactericide delivery to a specific model bacterium [60]. Antibacterial immunoliposomes were prepared using covalently bound antibody, extended to the cell surface of the bacterium Streptococcus oralis and chlorhexidine and triclosan were incorporated as the bactericides. For short exposure times to the biofilms, several times enhanced growth inhibition of $S$. oralis was obtained with immunoliposomes when compared to free bactericides.

Variable results have been reported in regard to relation between the surface charge of the liposomes and their effect on biofilms, most likely due to the differences between test methods used in the studies. Nguyen et al. [61] have reported that negatively charged liposomes, specifically targeting for the teeth, appeared to be the most suitable for use in the oral cavity because these liposomes were found to be the least reactive with the components of parotid saliva. On the other side, Sugano et al [62] have investigated the behavior of cationic liposomes on $S$. mutans in planktonic cells and biofilms and they reported that cationic liposomes have higher affinity not only to oral bacterial cells, but also biofilms than conventional liposomes. It was demonstrated microscopically that cationic liposomes interacted with the negative charge on the bacterial surface and penetrated the deep layers of biofilms.

Lectin-conjugated liposomes were prepared using wheat germ agglutinin (WGA) to serve as bioadhesive drug carrier that can rapidly bind to oral epithelial cells within minutes, and stay on the cells to provide sustained, localized drug release for the management of oral ulcerative lesions and other related complications [63]. A significant reduction in oral cell damage was obtained when the bacterially infected cells were treated with amoxicillin-loaded WGA liposomes compared to the untreated controls.

Erjavec et al [64] have investigated liposome formulations of varying composition and size to identify a suitable carrier for drug delivery to oral mucosal lesions by assessing the effects of a hyperaemic drug on the oral mucosa using in vivo EPR oximetry. They have reported that multi-lamellar liposomes made from hydrogenated soy lecithin appeared to be the most appropriate for local drug delivery to oral mucosa.

Liposome formulations have been widely investigated for treatment of periodontitis. It was shown in vivo that local delivery of liposome-encapsulated superoxide dismutase and catalase suppressed periodontal inflammation in experimentally induced periodontitis beagle dogs [65]. As an adjunctive treatment for chronic periodontitis, liposome formulation for an antimicrobial drug, minocycline was developed and investigated in vitro on murine macrophages (ANA1) [66]. Liposomes were shown to have stronger and longer inhibition effect on LPS-stimulated TNF-a secretion of macrophages cell when compared to that of solution of the drug.

pH-responsive quaternary ammonium chitosan (TMC) - liposome formulations loaded with doxycycline were developed for periodontal treatment [67]. The periodontitis healing capacity of the developed formulations was evaluated in rats. The formulations showed antimicrobial activity against $P$. gingivalis and Prevotella intermedia, strong inhibition on biofilm formation and prevented alveolar bone absorption in vivo.

Periodontal therapy usually requires also local anesthesia. A liposomal lidocaine/prilocaine, thermosetting anesthetic gel formulation delivered into periodontal pocket was investigated for pain control during scaling and root planing (anti-infective periodontal therapy) in 40 volunteers with moderate to severe chronic periodontitis [68]. It was reported that the intra-pocket anesthetic gel would be a good option for anxious patients, or those who have a fear of needles.

\section{Micelles}

Micelles are self-assembling colloidal systems obtained by the aggregation block or graft amphiphilic copolymers [69]. Micelles have found applications in dentistry for a targeted - delivery of antimicrobials to the tooth surface against biofilm formation. Chen et al [70], have used alendronate terminated Pluronic copolymers to prepare triclosan-loaded tooth-binding micelles and demonstrated that micelles were able to inhibit initial biofilm growth of $S$. mutans. The use of alendronate as a binding moiety, however, has raised concerns on the safety of these tooth-binding micelles therefore, the same group has replaced alendronate with diphosphoserine and conjugated it to the chain termini of Pluronic P123 and combined it with another biodegradable tooth-binding moiety, pyrophosphate (PPi) [71]. Tooth-binding potential and binding stability as well as anti-biofilm activity against $S$. mutans of the developed micelles were found to be significant. Recently a multifunctional matrix for the treatment of periodontitis and enhancement of regeneration of the periodontal tissue was prepared from vitamin E containing hydrogel made of alginate and gelatin, and doxycycline $\mathrm{HCl}$ containing methoxy poly(ethylene glycol)-block-polycaprolactone micelles [72]. A sustained drug release and enhanced antimicrobial activity were observed against $E$. coli and $S$. aureus.

\section{Hydrogels}


Hydrogels have a three-dimensional porous and interconnected structures composed of hydrophilic, cross-linked macromolecules that absorb water, aqueous solutions, or physiological fluids, but remain insoluble due to their network structure $[73,74]$. They provide a biocompatible microenvironment for cell attachment and proliferation, and possess many unique advantages on the targeted delivery systems for hydrophilic and hydrophobic agents and other biomolecules. Localized application is possible with hydrogels and they can be tailored to release the drug for a long time by controlling the hydrogel architectures, network pores, and gelation mechanisms (physical and chemical gelation). Synthetic (poly(hydroxyethyl methacrylate) (polyHEMA, PHEMA); polyethylene glycol and derivatives, poly(vinyl alcohol), polyvinylpyrrolidone, polyimide, polyacrylate, polyurethane) [75] and natural (chitosan, alginate, collagen, gelatin etc.) [76] polymers have been used for preparation of hydrogels. Most of these polymers exert also adhesive properties which enables a longer retention of the system on application site. Hydrogels have found applications in dentistry for regenerative therapies to provide recovery of the function of tissues lost due to oral and dental pathologies of infection as well as traumatic and neoplastic origin [77-79]. Furthermore, various hydrogel formulations have been used for treatment of oral lesions and also for delivery of antimicrobials, anaesthetics, antiinflammatory drugs [80-86]. Our group has investigated gel formulations based on chitosan, which is a material widely investigated in dental field both for its bioactive properties such as wound healing, tissue regeneration, antimicrobial and as a biocompatible, bioadhesive biopolymer for delivery of drugs, especially the anti-inflammatory and antimicrobial molecules [79, 87]. Chitosan gel itself has been shown to exhibit antimicrobial activity against various dental patogens [88]. Antimicrobial activity was found to depend on the properties of the chitosan used (source -animal or non-animal, molecular weight, solubility, degree of deacetylation etc.) as well as the type of the strains tested. Furthermore, when incorporated with various antimicrobial drugs such as chlorhexidine [82, 89], nystatin [90], moxifloxacin [91], metronidazole [81] and anti-inflammatory drug such as atorvastatin [80,92], the effect of the drug was found to be enhanced in presence of chitosan, besides the improved retention time and prolonged drug release. Chitosan gel itself has also been shown in vivo in human to be promising for periodontal tissue regeneration [93].

Hydrogels for the anesthetic drug lidocaine hydrochloride were prepared for buccal application using chitosan glutamate, or its binary mixture with glycerin. The anesthetic activity of mucoadhesive hydrogels was assessed in healthy volunteers in comparison to commercial semisolid formulations. Prolonged release of drug, which resulted in local anesthetic activity lasting for 20 to $30 \mathrm{~min}$ upon application was obtained. The developed hydrogels were suggested as potential delivery system reducing the pain symptoms that characterize aphthosis and other mouth diseases [94].

Hydrogels exert appropriate syringeability properties which makes it suitable for administration into periodontal pocket [95]. The injectable thermosensitive hydrogels have gained more attention, especially for unapproachable periodontal pockets. An injectable thermogel system for the treatment of oral mucosarelated ulcers was developed by Luo et al [96]. These thermogels were formed from a series of chitosan-based conjugates, composed of a chitosan backbone and synthetic side chains of thermosensitive poly(N-isopropylacrylamide) (PNIPAAM). Ulcer healing was investigated in vivo in rats and the antibacterial activity against Staphylococcus aureus as well as proliferation promotion, hemostasis effect of the developed formulation was demonstrated. Ji et al [97] have developed a thermosensitive hydrogel based on chitosan, quaternized chitosan and $\beta$-glycerophosphate loaded with $0.1 \% \mathrm{w} / \mathrm{w}$ chlorhexidine. Higher antimicrobial activity against $P$. gingivalis and Prevotella intermedia was obtained with gels prepared using quaternized chitosan when compared to that with chitosan.

A thermo-reversible poly-isocyanopeptide (PIC), which is a water soluble polymer forming a gel at very low polymer concentrations with good injectability properties, and has a sol-gel transition temperature of $15-18{ }^{\circ} \mathrm{C}$ [98], was investigated as a hydrogel for delivery of doxycycline and/or lipoxin A 4 for antimicrobial and anti-inflammatory treatment [99]. The PIC hydrogel facilitated the drug for around 4 days in vitro. When applied in dogs, local or systemic adverse effects were observed. The subgingival bacterial load and pro-inflammatory interleukin-8 level were shown to reduce with the hydrogel formulations. Gingival clinical attachment was improved when compared to mechanical debridement.

Dong et al [100] have incorporated metronidazole loaded microcapsules into a poly(vinyl alcohol) injectable hydrogel by dynamic covalent bonding and ionic interaction through a 4-carboxyphenylboronic acid bridge. The developed formulation exhibited desirable antibacterial activity against $P$. gingivalis and Fusobacterium nucleatum for 1 week period on the rats.

Hydrogels have been used also to deliver antimicrobial peptides (AMPs), which are one of the most well-studied classes of biofilm eradication agents [101, 102]. AMPs are a diverse group of host-defense molecules that include defensins, cathelicidins, histatins, neuropeptides, peptide hormones, and many other proven and putative peptides. In the oral cavity, the AMPs are produced by the salivary glands and the oral epithelium [103]. AMPs are effective defensive weapons and have been shown to modify cellular functions such as chemotaxis, apoptosis, gene transcription and cytokine production. Further, they play role in stimulation of wound healing and angiogenesis. Due to their antibacterial, anti-inflammatory and/or immune modulatory actions, they are used to control oral infections [104-110]. Sani et al [84] have developed a hydrogel based on a visible-light-activated naturally derived polymer (gelatin) and an antimicrobial peptide (AMP) for treatment of peri-implant diseases. An enhanced antimicrobial activity against $P$. gingivalis was obtained with the gels.

In oral mucosal conditions related to immunological pathogenesis, clinical studies have shown that topical immunomodulators such as cyclosporine, tacrolimus and pimecrolimus are also effective when compared to the steroids which are the conventionally used drugs [111-116]. In order to enhance their activities, these immunomodulators were incorporated into bioadhesive gels. For the treatment of oral lichen planus, clobetasol and cyclosporin adhesive gels based hydroxyethyl cellulose were applied twice a day on dried lesions for two months and significant healing was observed with the gels [117].

Currently, there are commercially available products based on hydrogels. A two syringe mixing system (Atridox) is a subgingival controlled-release product composed of the syringe A: Atrigel ${ }^{\circledR}$ Delivery System, which is a bioabsorbable, flowable polymeric formulation composed of $36.7 \%$ poly(DLlactide) (PLA) dissolved in $63.3 \% \mathrm{~N}$-methyl-2-pyrrolidone (NMP) and syringe B containing doxycycline hyclate [118]. Upon contact with the crevicular fluid, the liquid product solidifies and then allows for controlled release of drug for a period of 7 days. In addition, numerous gel formulations of metronidazole are also available on the market. 
Materials in nano size and drug-incorporated nanoparticles as well as their combination have found wide applications in dentistry for prevention, diagnosis, therapeutic, restoration and tissue regeneration purposes [52, 119-121].

Metallic nanoparticles such as silver, gold and zinc oxide due to their broad-spectrum antibacterial activity have been used to eliminate the biofilms in the oral cavity [122-127]. The large surface area and high charge density of these nanoparticles enable them to interact with the negatively-charged surface of bacterial cells to a greater extent resulting in enhanced antimicrobial activity. In order to enhance the antimicrobial activity, these metals have been combined with other antimicrobial agents such as chlorhexidine [128]. Recently, the antimicrobial efficacy of silver and gold nanoparticles with diode laser was investigated against $S$. mutans in teeth sample, and the greatest reduction in colony-forming units (CFU) was observed with the combination of silver nanoparticles with diode laser group [129].

Metallic nanoparticles combined with polymers or coated onto biomaterial surfaces have been shown to exhibit superior antimicrobial properties in the oral cavity [128, 130, 131]. Besides silver, gold and zinc oxide, bismuth subsalicylate nanoparticles have also been shown to inhibit the growth of several periodontal pathogens including A. actinomycetemcomitans, C. gingivalis, and P. gingivalis [132]

Further, mesoporous silica nanoparticles, which have a porous structure with large surface area, have been investigated as anti-biofilm agents [133, 134]. When combined with another antimicrobial such as chlorhexidine, antibacterial activity against S. mutans, F. nucleatum, A. actinomycetemcomitans and $P$. gingivalis was shown to be enhanced [135].

Recently, graphene family nanomaterials, due to their superior mechanical, chemical, and biological properties, have gained great attention in dentistry. Graphene oxide (GO), as the derivative of graphene, was investigated for its antimicrobial property against various dental pathogens including $S$. mutans, Fusobacterium nucleatum, P. gingivalis, and GO nanosheets were reported to be highly effective in inhibiting the growth of dental pathogens [136]. It was also shown by transmission electron microscopy that the cell wall and membrane of bacteria lost their integrity and the intracellular contents leaked out after they were treated by GO. Furthermore, graphene oxide (GO) has been widely investigated as a nanodelivery system for variety of drugs [137, 138], which makes it a promising material for treatment of infections in the oral cavity.

In the past decade, the application of antimicrobial photodynamic therapy (aPDT) on oral infectious diseases has attracted great interest. The bacteria can be killed when induced with light in presence of a sensitizing agent, by means of generation of cytotoxic, reactive oxygen species (ROS) [139]. There are a number of sensitizers that interact with bacterial cell and generate ROS such as methylene blue, erythrosine, indocyanine green, eosin-Y, psoralen, toluidine blue ortho [101]. Erythrosine has been applied with white light (500-650 nm) which successfully killed S. mutans and inhibited biofilm formation [140]. A dental light with haematoporphyrin sensitizer was investigated against S. mutans, A. actinomycetemcomitans and E. faecalis and it was found that the sensitizer can penetrate gram-positive bacteria cell, whilst by A. actinomycetemcomitans, the sensitizer is taken up in presence of 10\% EDTA [141]. Furthermore, dental LEDs with blue light absorbing photosensitizer were demonstrated to disrupt $E$. faecalis biofilm depending on the concentration of sensitizer [142]. However, due to the hydrophobic characteristics of the photosensitizers, aPDT was not very effective on the viability of biofilms, hence, nanomaterials (metal and metal oxide nanoparticles) or polymeric nanoparticles have been used in order to enhance the antimicrobial performance of aPDT [143, 144].

The photosensitizer indocyanine green (ICG) was incorporated into chitosan nanoparticles and

A. actinomycetemcomitans ATCC 33384 strain was treated with these nanoparticles, which was excited with a diode laser [145]. The expression of rcpA gene which is involved in biofilm formation of $A$. actinomycetemcomitans was found to be significantly downregulated upon using nanoparticles for aPDT, indicating a promising approach for control of periodontal pathogens. Similarly, indocyanine green was incorporated into PLGA nanoparticles coated with chitosan for aPDT [146]. A significantly higher antibacterial activity against $P$. gingivalis was observed. De Freitas et al [147] have investigated the effect of aPDT on human dental plaque bacteria using methylene blue (MB)-loaded poly(lactic-co-glycolic) nanoparticles in a clinical pilot study with 10 adult human subjects with chronic periodontitis. Patients were treated either with ultrasonic scaling and scaling and root planing (US + SRP) or ultrasonic Scaling + SRP + aPDT with MB-nanoparticles. The clinical study demonstrated the safety of aPDT. At month three, more profound effect ( $28.82 \%)$ on gingival bleeding index was observed in ultrasonic SRP + aPDT group when compared to ultrasonic SRP.

In literature there are numerous studies on polymeric nanoparticles used to deliver drugs into oral cavity for treatment of oral infections [148-152]. Due to their versatile characteristics such as surface charge, dimension and hydrophobicity, it has been possible to prepare tailor-made polymeric nanoparticles for an enhanced local treatment of oral infections (see Tables 1-3)

\section{Microparticles}

Polymer-based microparticles have also been investigated to maintain therapeutic drug concentrations for longer periods of time for treatment of dental and mucosal infections in the oral cavity $[153,154]$. Numerous synthetic (e.g.PLGA) and natural polymers (e.g. chitosan) have been successfully used in preparation of microparticles for drug delivery [155]. Due to its own antimicrobial activity, chitosan alone in microparticle form has also been investigated. Kawatika et al [156] have compared the effect of chitosan aqueous dispersion and microparticles on mature biofilms of $S$. mutans and demonstarted that chitosan in microparticle form reduced the bacterial viability and acidogenicity more effectively than the dispersions, thereby was more effective to control the growth of mature biofilms. Moura et al [157] have investigated the release of locally delivered doxycycline loaded PLGA microspheres in the periodontal pocket of patients with chronic periodontitis. The microspheres were demonstrated to provide sustained release after local administration, as an adjunct to nonsurgical periodontal therapy.

Currently, there is a commercially available subgingival sustained-release product (Arestin $\left.{ }^{\circledR}\right)$, which consists of minocycline hydrochloride incorporated microspheres prepared using bioresorbable polymer, poly (glycolide-co-dl-lactide). It is used in combination with scaling and root planing procedures to treat

Page 19/31 
patients with adult periodontitis [158].

\section{Strips / Fibers}

Strips and fibers composed of polymeric matrix have been used to deliver antimicrobials as an adjunct to mechanical treatment of periodontal disease [159]. They can be designed in appropriate dimension which allows practical insertion in periodontal pocket resulting in desirable clinical outcomes [160]. Various polymers and their combinations have been used to prepare strips and fiber for delivery of antimicrobials such as chlorhexidine, doxycycline, tetracycline, minocycline, metronidazole [161-163]. In early years, acrylic polymers have been widely used providing significant improvements in various clinical conditions by effective microbial eradication from the pockets [160]. However, due to several disadvantages such as being non-absorbable, removal required after therapy, which may impair the regenerating tissue at the site, other polymers such as cellulose derivatives (hydroxypropyl cellulose, hydroxypropyl methylcellulose, ethyl cellulose), polycaprolactone, polyhydroxybutyric acid, poly- methylmethacrylate, PLGA have been preferred over acrylic polymers.

Strips containing tetracycline hydrochloride or metronidazole $25 \%$ in polyhydroxybutyric acid as a biodegradable polymer matrix were evaluated in patients suffering from advanced periodontal disease [164]. The greatest response to therapy was observed with tetracycline hydrochloride strips inserted into periodontal pockets at four-day intervals for 16 days, compared with an untreated control group. Metronidazole strips or root-planing tended not to be as effective. The clinical improvement produced by each treatment was not maintained when treatment was terminated. A commercially available periodontal fiber (Actisite $\AA$ ) for periodontal pocket placement has been developed in $90 \mathrm{~s}$, which consists of a $23 \mathrm{~cm}$ monofilament of ethylene/vinyl acetate copolymer, $0.5 \mathrm{~mm}$ in diameter, impregnated with $25 \%$ tetracycline hydrochloride, providing continuous release of tetracycline for 10 days. It was demonstrated that the local delivery system was more effective than scaling and root planing (SRP) with respect to decreasing probing depth, increasing attachment level, and decreasing bleeding on probing (BOP) [165]. Later, the tetracycline strips were prepared with the identical polymer system to that of the fiber except for its physical shape and method of placement, and investigated in patients following administration singly or in multiples in conjunction with root planing, versus root planing alone, or to an untreated control [162]. It was concluded that multiple strips which fill the periodontal pocket were superior to a single strip in reducing BOP, and that use of locally delivered tetracycline was superior to SRP alone in decreasing probing depth.

The commercially available biodegradable local delivery system (PerioChip), which contains chlorhexidine gluconate in a biodegradable matrix of hydrolyzed gelatin (cross-linked with glutaraldehyde) developed by Steinberg et al [166] can also be considered as a strip with rectangular shape. Drug concentration was shown to remain above the minimum inhibitory concentration for more than $99 \%$ of periodontal pocket flora for up to nine days.

J Periodontol $\cdot$ January 2002

In recent years, nanostructured polymeric fibers have been developed in order to enhance the efficacy of the drugs at the application site [167]. The advantages of nanofibers involve large surface area, high porosity, high mechanical strength which make it a potential candidate for the application into the periodontal pocket [168]. Electrospinning technique is the mostly used method for the production of nanofibers, which allows adjustment of fiber size, drug loading and mechanical properties can be adjusted [169]. In a recent study, the antibacterial activity of chitosan nanofiber, cross-linked with tetracycline comprising polyvinyl alcohol (PVA) was evaluated and enhanced antibacterial activity against a wide range of periodontal pathogens was demonstrated [170]. Mahmoud et al [171] have developed polymeric electrospun fibers using PLGA, poly(L-lactic acid) (PLLA), and polycaprolactone (PCL) alone or blended with polyethylene oxide (PEO), incorporated with an antimicrobial peptide (BAR), and evaluated their safety and functionality against $P$. gingivalis/ $S$. gordonii biofilms. The most promising formulation was found to be PLGA:PEO providing a sustained drug release and a dose-dependent inhibition of biofilm formation.

\section{Films And Wafers}

Films containing antimicrobials and anti-inflammatory drugs have been designed for treatment of oral infections [172, 173]. Film formulations can be applied on the oral mucosa as well as into the periodontal pockets. They are thin and flexible, and particularly, mucoadhesive films can resist to physiological conditions in the oral cavity [174]. Numerous synthetic or natural polymers have been investigated to develop mucoadhesive films with one or more layers, or films based on stimuli responsive hydrogel. Chitosan is one of the most investigated polymers for preparation of films. Due to its bioadhesive properties, it can retain on the application site in prolonged periods of time and also exerts synergetic effect due to it antimicrobial activity [89, 175]. Recently, a two layerpolymeric film was prepared using a polymeric gel-like blend (including chitosan, HPMC, methocel at various ratios), with the basal layer (with lidocaine hydrochloride for a faster release than the apical layer with benzydamine $\mathrm{HCl}$ and $\mathrm{N}$-acetyl-cysteine [176]. The single patient study showed that the association of polymers with the addition of analgesics, anti-inflammatories, and mucolytics promoted the reduction of inflammation, tumefaction, and an erythematous halo with significant mucosal regeneration in 30 days.

Exerting similar properties to that of films, wafers have been also investigated for local delivery to the oral cavity. The advantage of wafers of films is reported to be low residual moisture and increased drug loading [177]. Recently our group has developed monolayer and bilayered mucoadhesive film and wafer formulations as local drug delivery platforms for treatment of oral infections, using chitosan and hydroxypropyl methylcellulose (HPMC) [178]. Cefuroxime axetil (CA) was used as the model drug. Antimicrobial activity was evaluated against $E$. coli and $S$. aureus. HPMC based formulations were found to disintegrate within less than 30 min whereas chitosan based formulations remained intact up to $6 \mathrm{~h}$. Significantly higher drug release was obtained with wafer formulations. Antimicrobial activity was found to increase in presence of chitosan, and HPMC was also observed to contribute to antimicrobial activity. Bilayered wafer formulation, with adhesive chitosan backing layer and HPMC based drug loaded layer is suggested as a promising local delivery system for treatment of the infections in the oral cavity.

\section{Conclusion And Future Perspectives}


The mouth is said to be the gateway to one's overall health, because the oral cavity may exhibit manifestations of underlying systemic infectious or autoimmune, hematologic, endocrine, and neoplastic related diseases, and serve as an indicator of overall health. On the other hand, oral cavity can act also as the site of origin for dissemination of pathogenic organisms to distant body sites, especially in immunocompromised hosts such as patients suffering from diabetes or rheumatoid arthritis or receiving immunosuppressive treatment. The oral microbiome encompasses a highly diverse microbiota, consisting of over 700 microorganisms, including bacteria, fungi, and viruses. In order to maintain the oral health, it is important to diagnose precisely the underlying local or systemic condition of the oral diseases in order to take the right actions for prevention and treatment. Appropriate drugs and delivery systems are crucial to reduce /eliminate the local as well as the systemic complications related to oral infections. Currently, there are numerous drugs available for preventive and palliative therapies, which reduce the infection incidence but cannot adequately eliminate the infection. Topical use of antivirals and antimicrobials have been successfully used for treatment of oral infections however there are still some obstacles to be addressed related to the drugs used as well as the delivery systems. Antimicrobial resistance has emerged as a huge challenge to the effective treatment of infectious diseases with antibiotics. Owing to the challenges faced with discovery of a new drug and the limited number of new classes of antibiotics, researchers have focused more on non-conventional approaches that could serve as alternatives to antibiotics. The alternative approaches to antibiotics include immunomodulators, competitive exclusion of pathogenic bacteria via probiotics and their combination, antimicrobial peptides, and photoactivatable agents. Another widely investigated approach against antimicrobial resistance is use of nanomaterials either as the active agent (e.g. silver or gold nanoparticles, nanographene, zinc oxide) or for drug delivery (antibiotics or above-mentioned alternate compounds). Nano delivery systems have the potential to deliver the antibiotic payload into the infected cells, therefore enhancing penetration and release of antibiotics inside the infected cells. By this means, they can reduce the antibiotic overuse and help prevent antimicrobial resistance. Furthermore, nano systems have the potential to treat biofilm-forming pathogen infections by acting as a protective coat, shielding against interactions of drug by biofilm compartments and resident enzymes. Nevertheless, oral cavity is a complex environment for local drug delivery due to its distinct characteristics such as different epithelial structure (keratinized or non-keratinized, thickness etc) in different regions, continuous secretion of saliva, movement of the tongue and swallowing. Specialized delivery systems are required to deliver the drug in the desired period of time, resisting the physiological conditions of the oral cavity and avoiding the removal of the drug from the application site. Furthermore, for delivery to certain regions in the oral cavity such as periodontal pocket and tooth, systems with certain shape and dimension are required.

Currently, there are numerous products available on the market, which are mostly based on conventional dosage forms and aimed for inhibition of bacterial growth and biofilm formation, for reducing inflammation or for tissue regeneration. On the other side, many promising results have been reported for prevention and treatment of oral infections with the newly developed advanced systems, particularly, with those that apply nanotechnology and/or use novel compounds alternative to those currently used, however, up to date very few have reached to the market. More studies in human are needed to prove the safety and efficacy of these systems, so that they can be available for routine clinical applications. In regard to manufacturing of these systems, commercial scaleup may pose some problems due to their complexities however the delivery market opportunities are plentiful. There are indeed some exciting developments within topical delivery into the oral cavity. For future, the most lacking research area appears to be the development of vaccines for prevention of oral infections. This is indeed an important aspect on which the researchers should focus more.

\section{Declarations}

\section{Ethical statement:}

Ethics approval and consent to participate:

not applicable

Consent for publication:

All authors have the consent for publication.

\section{Competing interests:}

There is no conflict of interest with any person or company.

\section{Funding:}

not applicable

\section{Authors' contributions:}

Authors have contributed equally in this manuscript.

\section{Acknowledgements:}

not applicable 


\section{References}

1. Lamont RJ, Koo H, Hajishengallis G. The oral microbiota: dynamic communities and host interactions. Nat Rev Microbiol. 2018;16(12):745-59. doi:https://doi.org/10.1038/s41579-018-0089-x.

2. WHO. Oral health. In: Newsroom. 2020. https://www.who.int/news-room/fact-sheets/detail/oral-health. Accessed 4 Jan 2021.

3. Moving together to build a healthier world. In: Political Declaration of the High-level Meeting on Universal Health Coverage. 2019. https://www.un.org/pga/73/wp-content/uploads/sites/53/2019/07/FINAL-draft-UHC-Political-Declaration.pdf. Accessed 4 Jan 2021.

4. Santosh ABR, Muddana K. Viral infections of oral cavity. J Family Med Prim Care. 2020;9(1):36. doi:https://doi.org/10.4103/jfmpc.jfmpc_807_19.

5. Gondivkar S, Gadbail A, Sarode GS, Sarode SC, Patil S, Awan KH. Infectious diseases of oral cavity. Dis Mon. 2019;65(6):164. doi:https://doi.org/10.1016/j.disamonth.2018.09.008.

6. Donà MG, Spriano G, Pichi B, Rollo F, Laquintana V, Covello R et al. Human papillomavirus infection and p16 overexpression in oropharyngeal squamous cell carcinoma: a case series from 2010 to 2014. Future Microbiol. 2015;10(8):1283-91. doi:https://doi.org/10.2217/FMB.15.55.

7. Asai D, Nakashima H. Pathogenic viruses commonly present in the oral cavity and relevant antiviral compounds derived from natural products. Medicines. 2018;5(4):120. doi:https://doi.org/10.3390/medicines5040120.

8. Scott DA, Coulter WA, Biagioni PA, O'Neill HO, Lamey PJ. Detection of herpes simplex virus type 1 shedding in the oral cavity by polymerase chain reaction and enzyme-linked immunosorbent assay at the prodromal stage of recrudescent herpes labialis. J Oral Pathol Med. 1997;26(7):305-9. doi:https://doi.org/10.1111/j.1600-0714.1997.tb00220.x.

9. Rautava J, Syrjänen S. Human papillomavirus infections in the oral mucosa. J Am Dent Assoc. 2011;142(8):905-14. doi:https://doi.org/10.14219/jada.archive.2011.0297.

10. Stoopler ET, Balasubramanlam R. Topical and systemic therapies for oral and perioral herpes simplex virus infections. J Calif Dent Assoc. 2013;41(4):259.

11. Hammer KD, Dietz J, Lo TS, Johnson EM. A systematic review on the efficacy of topical acyclovir, penciclovir, and docosanol for the treatment of herpes simplex labialis. Dermatology. 2018.

12. WHO. Coronavirus disease 2019 (COVID-19) Situation Report-51, https://www.who.int/docs/default-source/coronaviruse/situation-reports/20200311sitrep-51-covid-19.pdf. Accessed 7 Jan 2021.

13. de Wit E, van Doremalen N, Falzarano D, Munster VJ. SARS and MERS: recent insights into emerging coronaviruses. Nat Rev Microbiol. 2016;14(8):52334. doi:https://doi.org/10.1038/nrmicro.2016.81.

14. Hu B, Guo H, Zhou P, Shi Z-L. Characteristics of SARS-CoV-2 and COVID-19. Nat Rev Microbiol. 2020:1-14. doi:https://doi.org/10.1038/s41579-020-004597.

15. Xu H, Zhong L, Deng J, Peng J, Dan H, Zeng X et al. High expression of ACE2 receptor of 2019-nCoV on the epithelial cells of oral mucosa. Int J Oral Sci. 2020;12(1):8. doi:https://doi.org/10.1038/s41368-020-0074-x.

16. Wang C, Wu H, Ding X, Ji H, Jiao P, Song H et al. Does infection of 2019 novel coronavirus cause acute and/or chronic sialadenitis? Med Hypotheses. 2020:109789. doi:https://doi.org/10.1016/j.mehy.2020.109789.

17. Meng L, Hua F, Bian Z. Coronavirus Disease 2019 (COVID-19): Emerging and Future Challenges for Dental and Oral Medicine. J Dent Res. 2020;99(5):4817. doi:https://doi.org/10.1177/0022034520914246.

18. Martin Carreras-Presas C, Amaro Sanchez J, Lopez-Sanchez AF, Jane-Salas E, Somacarrera Perez ML. Oral vesiculobullous lesions associated with SARSCoV-2 infection. Oral Dis. 2020. doi:10.1111/odi.13382.

19. Amorim dos Santos J, Normando A, Carvalho da Silva R, Acevedo A, De Luca Canto G, Sugaya N et al. Oral manifestations in patients with COVID-19: a living systematic review. J Dent Res. 2020:0022034520957289. doi:https://doi.org/10.1177/0022034520957289.

20. Iranmanesh B, Amiri R, Zartab H, Aflatoonian M. Oral manifestations of COVID-19 disease: A review article. Dermatol Ther. 2020. doi:https://doi.org/10.1111/dth.14578.

21. Xu J, Li Y, Gan F, Du Y, Yao Y. Salivary glands: potential reservoirs for COVID-19 asymptomatic infection. J Dent Res. 2020:0022034520918518.

22. Xu R, Cui B, Duan X, Zhang P, Zhou X, Yuan Q. Saliva: potential diagnostic value and transmission of 2019-nCoV. Int J Oral Sci. 2020;12(1):1-6. doi:https://doi.org/10.1038/s41368-020-0080-z.

23. Díaz Rodríguez M, Jimenez Romera A, Villarroel M. Oral manifestations associated with COVID-19. Oral Dis. 2020. doi:https://doi.org/10.1111/odi.13555|

24. Krishnan PA. Fungal infections of the oral mucosa. Indian J Dent Res. 2012;23(5):650.

25. Telles DR, Karki N, Marshall MW. Oral fungal infections: diagnosis and management. Dent Clin. 2017;61(2):319-49. doi:http://dx.doi.org/10.1016/j.cden.2016.12.004.

26. Garcia-Cuesta C, Sarrion-Pérez M-G, Bagán JV. Current treatment of oral candidiasis: A literature review. J Clin Exp Dent. $2014 ; 6(5): e 576$. doi:http://dx.doi.org/10.4317/jced.51798.

27. Quindós G, Gil-Alonso S, Marcos-Arias C, Sevillano E, Mateo E, Jauregizar N et al. Therapeutic tools for oral candidiasis: Current and new antifungal drugs. Med Oral Patol Oral Cir Bucal. 2019;24(2):e172. doi:http://dx.doi.org/doi:10.4317/medoral.22978.

28. Nowak A, Christensen JR, Mabry TR, Townsend JA, Wells MH. Pediatric Dentistry-E-Book: Infancy through Adolescence. Elsevier Health Sciences; 2018.

29. Aas JA, Paster BJ, Stokes LN, Olsen I, Dewhirst FE. Defining the normal bacterial flora of the oral cavity. J Clin Microbiol. 2005;43(11):5721-32. doi:https://doi.org/10.1128/JCM.43.11.5721-5732.2005.

Page 22/31 
30. Levi ME, Eusterman VD. Oral infections and antibiotic therapy. Otolaryngol Clin North Am. 2011;44(1):57-78. doi:https://doi.org/10.1016/j.otc.2010.10.003.

31. Dahlen G. Bacterial infections of the oral mucosa. Periodontol 2000. 2009;49:13-38. doi:https://doi.org/10.1111/j.1600-0757.2008.00295.x.

32. Jiao Y, Tay FR, Niu L-n, Chen J-h. Advancing antimicrobial strategies for managing oral biofilm infections. Int J Oral Sci. 2019;11(3):1-11. doi:https://doi.org/10.1038/s41368-019-0062-1.

33. Spratt D, Pratten J. Biofilms and the oral cavity. Rev Environ Sci Biotechnol. 2003;2(2-4):109-20. doi:https://doi.org/10.1023/B:RESB.0000040466.82937.df.

34. Newman MG, Takei HH, Klokkevold PR. Carranza's clinical periodontology.12th ed. St Louis: Elsevier; 2015.

35. Chi AC, Neville BW, Krayer JW, Gonsalves WC. Oral manifestations of systemic disease. Am Fam Physician. 2010;82(11):1381-8.

36. Rivera-Hidalgo F, Stanford TW. Oral mucosal lesions caused by infective microorganisms I. Viruses and bacteria. Periodontol 2000. 1999;21(1):106-24. doi: https://doi.org/10.1111/j.1600-0757.1999.tb00171.x.

37. Verderosa AD, Totsika M, Fairfull-Smith KE. Bacterial biofilm eradication agents: a current review. Front Chem. $2019 ; 7: 824$. doi:https://doi.org/10.3389/fchem.2019.00824.

38. Shastry SP, Sanjay C, Kaul R, Mahima V, Doggalli N. Topical drug delivery: An essential aid in the management of oral diseases. J Adv Clin Res Insights. 2015;2(6):269-75. doi:https://doi.org/10.15713/ins.jcri.92.

39. Collins $L$, Dawes $C$. The surface area of the adult human mouth and thickness of the salivary film covering the teeth and oral mucosa. $J$ Dent Res. 1987;66(8):1300-2. doi:https://doi.org/10.1177/00220345870660080201.

40. Frenkel ES, Ribbeck K. Salivary mucins in host defense and disease prevention. J Oral Microbiol. 2015;7(1):29759. doi:https://doi.org/10.3402/jom.v7.29759.

41. Fenton OS, Olafson KN, Pillai PS, Mitchell MJ, Langer R. Advances in biomaterials for drug delivery. Advanced Materials. 2018;30(29):1705328.

42. George A, Shah PA, Shrivastav PS. Natural biodegradable polymers based nano-formulations for drug delivery: A review. Int J Pharm. 2019;561:244-64. doi:https://doi.org/10.1016/j.ijpharm.2019.03.011.

43. Bruschi ML, de Freitas O. Oral bioadhesive drug delivery systems. Drug Dev Ind Pharm. 2005;31(3):293-310. doi:https://doi.org/10.1081/DDC-52073.

44. Zięba M, Chaber P, Duale K, Martinka Maksymiak M, Basczok M, Kowalczuk M et al. Polymeric Carriers for Delivery Systems in the Treatment of Chronic Periodontal Disease. Polymers. 2020;12(7):1574. doi:https://doi.org/10.3390/polym12071574.

45. Parhi R. Drug delivery applications of chitin and chitosan: a review. J Environmental Chemistry Letters. 2020:1-18. doi:https://doi.org/10.1007/s10311020-00963-5.

46. Hua S. Advances in nanoparticulate drug delivery approaches for sublingual and buccal administration. Front Pharmacol. 2019;10. doi:https://doi.org/10.3389/fphar.2019.01328.

47. Colton MB, Ehrlich E. Bactericidal effect obtained by addition of antibiotics to dental cements and direct filling resins. J Am Dent Assoc. 1953;47(5):52431. doi:https://doi.org/10.14219/jada.archive.1953.0206.

48. Greenstein G, Polson A. The role of local drug delivery in the management of periodontal diseases: a comprehensive review. J Periodontol. 1998;69(5):507-20. doi:https://doi.org/10.1902/jop.1998.69.5.507.

49. Soskolne W, Heasman P, Stabholz A, Smart G, Palmer M, Flashner M et al. Sustained local delivery of chlorhexidine in the treatment of periodontitis: A multi-center study. J Periodontol. 1997;68(1):32-8. doi:https://doi.org/10.1902/jop.1997.68.1.32.

50. Sander L, Frandsen EVG, Arnbjerg D, Warrer K, Karring T. Effect of local metronidazole application on periodontal healing following guided tissue regeneration. Clinical findings. J Periodontol. 1994;65(10):914-20. doi:https://doi.org/10.1902/jop.1994.65.10.914.

51. Graca M, Watts T, Wilson R, Palmer R. A randomized controlled trial of a $2 \%$ minocycline gel as an adjunct to non-surgical periodontal treatment, using a design with multiple matching criteria. J Clin Periodontol. 1997;24(4):249-53. doi:https://doi.org/10.1111/j.1600-051X.1997.tb01838.X.

52. Fabio Oliveira de Sousa F, Ferraz C, de Santiago Nojosa J, Yamauti M. Nanotechnology in dentistry: drug delivery systems for the control of biofilmdependent oral diseases. Curr Drug Deliv. 2014;11(6):719-28.

53. Torchilin VP. Recent advances with liposomes as pharmaceutical carriers. Nat Rev Drug Discov. 2005;4(2):145-60. doi:https://doi.org/10.1038/nrd1632.

54. Gregoriadis G, Perrie Y. Liposomes. Encyclopedia of Life Sciences (ELS). JohnWiley \& Sons, Ltd: Chicheste. 2010.

55. Mezei M, Gulasekharam V. Liposomes-a selective drug delivery system for the topical route of administration. Life Sci. 1980;26:1473-7. doi:https://doi.org/10.1111/j.2042-7158.1982.tb04767.x.

56. Mezei M. Liposomes a selective drug delivery system for the topical route of administration: gel dosage. J Pharm Pharmacol. 1982;34:473-4. doi:https://doi.org/10.1016/0024-3205(80)90268-4.

57. Harsanyi B, Hilchie J, Mezei M. Liposomes as drug carriers for oral ulcers. J Dent Res. 1986;65(9):1133-41. doi:https://doi.org/10.1177/00220345860650090501.

58. Jones MN, Francis SE, Hutchinson FJ, Handley PS, Lyle IG. Targeting and delivery of bactericide to adsorbed oral bacteria by use of proteoliposomes. Biochim Biophys Acta Biomembr. 1993;1147(2):251-61. doi:https://doi.org/10.1016/0005-2736(93)90010-W.

59. Hill KJ, Kaszuba M, Creeth JE, Jones MN. Reactive liposomes encapsulating a glucose oxidase-peroxidase system with antibacterial activity. Biochim Biophys Acta Biomembr. 1997;1326(1):37-46. doi:https://doi.org/10.1016/S0005-2736(97)00007-2.

60. Robinson AM, Creeth JE, Jones MN. The use of immunoliposomes for specific delivery of antimicrobial agents to oral bacteria immobilized on polystyrene. J Biomater Sci Polym Ed. 2000;11(12):1381-93. doi:https://doi.org/10.1163/156856200744408.

Page 23/31 
61. Nguyen S, Hiorth M, Rykke M, Smistad G. The potential of liposomes as dental drug delivery systems. Eur J Pharm Biopharm. 2011;77(1):75-83. doi:https://doi.org/10.1016/j.ejpb.2010.09.010.

62. Sugano M, Morisaki H, Negishi Y, Endo-Takahashi Y, Kuwata H, Miyazaki T et al. Potential effect of cationic liposomes on interactions with oral bacterial cells and biofilms. J Liposome Res. 2016;26(2):156-62. doi:https://doi.org/10.3109/08982104.2015.1063648.

63. Wijetunge SS, Wen J, Yeh C-K, Sun Y. Lectin-conjugated liposomes as biocompatible, bioadhesive drug carriers for the management of oral ulcerative lesions. ACS Appl Bio Mater. 2018;1(5):1487-95. doi:https://doi.org/10.1021/acsabm.8b00425.

64. Erjavec V, Pavlica Z, Sentjurc M, Petelin M. In vivo study of liposomes as drug carriers to oral mucosa using EPR oximetry. Int J Pharm. 2006;307(1):1-8. doi:https://doi.org/10.1016/j.ijpharm.2005.09.016.

65. Petelin M, Pavlica Z, Ivanuša T, Šentjurc M, Skalerič U. Local delivery of liposome-encapsulated superoxide dismutase and catalase suppress periodontal inflammation in beagles. J Clin Periodontol. 2000;27(12):918-25. doi:https://doi.org/10.1034/j.1600-051x.2000.027012918.x.

66. Liu D, Yang P. Minocycline hydrochloride nanoliposomes inhibit the production of TNF-a in LPS-stimulated macrophages. Int J Nanomedicine. 2012;7:4769. doi:https://doi.org/10.2147/IJN.S34036.

67. Hu F, Zhou Z, Xu Q, Fan C, Wang L, Ren H et al. A novel pH-responsive quaternary ammonium chitosan-liposome nanoparticles for periodontal treatment. Int J Biol Macromol. 2019;129:1113-9. doi:https://doi.org/10.1016/j.ijbiomac.2018.09.057.

68. Moraes GS, Santos IBd, Pinto SCS, Pochapski MT, Farago PV, Pilatti GL et al. Liposomal anesthetic gel for pain control during periodontal therapy in adults: a placebo-controlled RCT. J Appl Oral Sci. 2020;28. doi:https://doi.org/10.1590/1678-7757-2019-0025.

69. Capretto L, Mazzitelli S, Colombo G, Piva R, Penolazzi L, Vecchiatini R et al. Production of polymeric micelles by microfluidic technology for combined drug delivery: application to osteogenic differentiation of human periodontal ligament mesenchymal stem cells (hPDLSCs). Int J Pharmaceut. 2013;440(2):195-206. doi:https://doi.org/10.1016/j.ijpharm.2012.07.057.

70. Chen F, Rice KC, Liu X-M, Reinhardt RA, Bayles KW, Wang D. Triclosan-loaded tooth-binding micelles for prevention and treatment of dental biofilm. Pharm Res. 2010;27(11):2356-64. doi:https://doi.org/10.1007/s11095-010-0119-5.

71. Chen F, Jia Z, Rice KC, Reinhardt RA, Bayles KW, Wang D. The development of dentotropic micelles with biodegradable tooth-binding moieties. Pharm Res. 2013;30(11):2808-17. doi:https://doi.org/10.1007/s11095-013-1105-5.

72. Isik G, Hasirci N, Tezcaner A, Kiziltay A. Multifunctional periodontal membrane for treatment and regeneration purposes. J Bioact Compat Polym. 2020;35(2):117-38. doi:https://doi.org/10.1177/0883911520911659.

73. Hoffman A. Hydrogel biomedical articles. Adv Drug Deliver Rev. 2002;54:3-12. doi:https://doi.org/10.1016/S0169-409X(01)00239-3.

74. Peppas N, Slaughter B, Kanzelberger M. Hydrogels. In Polymer Science: A Comprehensive Reference. Amsterdam: Elsevier; 2012. pp. 385-395. doi:https://doi.org/10.1016/B978-0-444-53349-4.00226-0.

75. Chai Q, Jiao Y, Yu X. Hydrogels for biomedical applications: their characteristics and the mechanisms behind them. Gels. 2017;3(1):6. doi:https://doi.org/10.3390/gels3010006.

76. Catoira MC, Fusaro L, Di Francesco D, Ramella M, Boccafoschi F. Overview of natural hydrogels for regenerative medicine applications. J Mater Sci Mater Med. 2019;30(10):115. doi:https://doi.org/10.1007/s10856-019-6318-7.

77. Yen A-H, Yelick P. Dental tissue regeneration-a mini-review. Gerontology. 2011;57(1):85-94. doi:https://doi.org/10.1159/000314530.

78. Alexandre Chisini L, Cristian Muniz Conde M, Grazioli G, Schmidt San Martin A, Fernando Demarco F. Bone, Periodontal and Dental Pulp Regeneration in Dentistry: A Systematic Scoping Review. Braz Dent J. 2019;30:77-95. doi:https://doi.org/10.1590/0103-6440201902053.

79. Şenel S, Aksoy EA, Akca G. Application of chitosan based scaffolds for drug delivery and tissue engineering in dentistry. Marine-Derived Biomaterials for Tissue Engineering Applications. Springer; 2019. p. 157-78.

80. Ozdogan Al, Ilarslan YD, Kosemehmetoglu K, Akca G, Kutlu HB, Comerdov E et al. In vivo evaluation of chitosan based local delivery systems for atorvastatin in treatment of periodontitis. Int J Pharm. 2018;550(1-2):470-6. doi:https://doi.org/10.1016/j.ijpharm.2018.08.058.

81. Akıncıbay H, Şenel S, Yetkin Ay Z. Application of chitosan gel in the treatment of chronic periodontitis. J Biomed Mater Res B Appl Biomater. 2007;80(2):290-6. doi:https://doi.org/10.1002/jbm.b.30596.

82. Ikinci G, Şenel S, Akincibay H, Kas S, Ercis S, Wilson CG et al. Effect of chitosan on a periodontal pathogen Porphyromonas gingivalis. Int J Pharm. 2002;235(1-2):121-7. doi:https://doi.org/10.1016/S0378-5173(01)00974-7.

83. Cicciù M, Fiorillo L, Cervino G. Chitosan use in dentistry: A systematic review of recent clinical studies. Mar Drugs. 2019;17(7):417. doi:https://doi.org/10.3390/md17070417.

84. Sani ES, Lara RP, Aldawood Z, Bassir SH, Nguyen D, Kantarci A et al. An antimicrobial dental light curable bioadhesive hydrogel for treatment of periimplant diseases. Matter. 2019;1(4):926-44. doi:https://doi.org/10.1016/j.matt.2019.07.019.

85. Hamed R, AbuRezeq Aa, Tarawneh O. Development of hydrogels, oleogels, and bigels as local drug delivery systems for periodontitis. Drug Dev Ind Pharm. 2018;44(9):1488-97. doi:https://doi.org/10.1080/03639045.2018.1464021.

86. Yang Z, Liang X, Jiang X, Guo J, Tao Y, Wang S et al. Development and evaluation of minocycline hydrochloride-loaded in situ cubic liquid crystal for intraperiodontal pocket administration. Molecules. 2018;23(9):2275. doi:https://doi.org/10.3390/molecules23092275.

87. Şenel S. Current status and future of chitosan in drug and vaccine delivery. React Funct Polym. 2019:104452. doi:https://doi.org/10.1016/j.reactfunctpolym.2019.104452.

88. Akca G, Ozdemir A, Oner ZG, Şenel S. Comparison of different types and sources of chitosan for the treatment of infections in the oral cavity. Res Chem Intermediat. 2018;44(8):4811-25. doi:https://doi.org/10.1007/s11164-018-3338-8.

Page 24/31 
89. Şenel S, Ikinci G, Kas S, Yousefi-Rad A, Sargon MF, Hincal AA. Chitosan films and hydrogels of chlorhexidine gluconate for oral mucosal delivery. Int J Pharm. 2000;193(2):197-203. doi:https://doi.org/10.1016/S0378-5173(99)00334-8.

90. Aksungur P, Sungur A, Unal S, Iskit AB, Squier CA, Şenel S. Chitosan delivery systems for the treatment of oral mucositis: in vitro and in vivo studies. $J$ Control Release. 2004;98(2):269-79. doi:https://doi.org/10.1016/j.jconrel.2004.05.002.

91. Azeran N, Zazali N, Timur S, Özdogan I, Ekizoglu M, Sheshala R et al. Moxifloxacin Loaded Chitosan Gel Formulations for the Treatment of Periodontal Diseases. J Polym Mater. 2017;34:157-70.

92. Ozdogan Al, Akca G, Şenel S. Development and in vitro evaluation of chitosan based system for local delivery of atorvastatin for treatment of periodontitis. Eur J Pharm Sci. 2018;124:208-16. doi:https://doi.org/10.1016/j.ejps.2018.08.037.

93. Boynuegri D, Ozcan G, Şenel S, Uc D, Uraz A, Ogus E et al. Clinical and Radiographic Evaluations of Chitosan Gel in Periodontal Intraosseous Defects: A Pilot Study. J Biomed Mater Res B. 2009;90b(1):461-6. doi:https://doi.org/10.1002/jbm.b.31307.

94. Pignatello R, Basile L, Puglisi G. Chitosan glutamate hydrogels with local anesthetic activity for buccal application. Drug deliv. 2009;16(3):176-81. doi:https://doi.org/10.1080/10717540902861267.

95. Wang B, Booij-Vrieling HE, Bronkhorst EM, Shao J, Kouwer PH, Jansen JA et al. Antimicrobial and anti-inflammatory thermo-reversible hydrogel for periodontal delivery. Acta Biomaterialia. 2020;116:259-67. doi:https://doi.org/10.1016/j.actbio.2020.09.018.

96. Luo Z, Xue K, Zhang X, Lim JY, Lai X, Young DJ et al. Thermogelling chitosan-based polymers for the treatment of oral mucosa ulcers. Biomater Sci. 2020;8(5):1364-79. doi:https://doi.org/10.1039/C9BM01754B.

97. Ji QX, Chen XG, Zhao QS, Liu CS, Cheng XJ, Wang LC. Injectable thermosensitive hydrogel based on chitosan and quaternized chitosan and the biomedical properties. J Mater Sci Mater Med. 2009;20(8):1603-10. doi:https://doi.org/10.1007/s10856-009-3729-x.

98. Zimoch J, Padial JS, Klar AS, Vallmajo-Martin Q, Meuli M, Biedermann T et al. Polyisocyanopeptide hydrogels: A novel thermo-responsive hydrogel supporting pre-vascularization and the development of organotypic structures. Acta biomater. 2018;70:129-39. doi:https://doi.org/10.1016/j.actbio.2018.01.042.

99. Wang B, Booij-Vrieling HE, Bronkhorst EM, Shao J, Kouwer PH, Jansen JA et al. Antimicrobial and anti-inflammatory thermo-reversible hydrogel for periodontal delivery. Acta Biomater. 2020;116:259-67. doi:https://doi.org/10.1016/j.actbio.2020.09.018.

100. Dong Z, Sun Y, Chen Y, Liu Y, Tang C, Qu X. Injectable Adhesive Hydrogel through a Microcapsule Cross-Link for Periodontitis Treatment. ACS Applied Bio Materials. 2019;2(12):5985-94. doi:https://doi.org/10.1021/acsabm.9b00912.

101. Allaker RP, Ian Douglas C. Non-conventional therapeutics for oral infections. Virulence. 2015;6(3):196-207. doi:https://doi.org/10.4161/21505594.2014.983783

102. Borro BC, Nordström R, Malmsten M. Microgels and hydrogels as delivery systems for antimicrobial peptides. Colloids Surf B. $2020: 110835$. doi:https://doi.org/10.1016/j.colsurfb.2020.110835.

103. Zasloff M. Antimicrobial peptides in health and disease. N Engl J Med. 2002;347(15):1199-. doi:https://doi.org/10.1038/415389a.

104. Niu JY, Yin IX, Wu WKK, Li Q-L, Mei ML, Hung CC. Antimicrobial peptides for the prevention and treatment of dental caries: A concise review. Arch Oral Biol. 2020:105022. doi:https://doi.org/10.1016/j.archoralbio.2020.105022.

105. Wattanarat O, Makeudom A, Sastraruji T, Piwat S, Tianviwat S, Teanpaisan R et al. Enhancement of salivary human neutrophil peptide 1-3 levels by probiotic supplementation. BMC Oral Health. 2015;15(1):19. doi:https://doi.org/10.1186/s12903-015-0003-0.

106. Lee J-K, Chang SW, Perinpanayagam H, Lim S-M, Park Y-J, Han SH et al. Antibacterial efficacy of a human $\beta$-defensin-3 peptide on multispecies biofilms. J Endod. 2013;39(12):1625-9. doi:https://doi.org/10.1016/j.joen.2013.07.035.

107. Zhou L, Wong HM, Zhang YY, Li QL. Constructing an Antibiofouling and Mineralizing Bioactive Tooth Surface to Protect against Decay and Promote SelfHealing. ACS Appl Mater Interfaces. 2019;12(2):3021-31. doi:https://doi.org/10.1021/acsami.9b19745.

108. Krzyściak W, Jurczak A, Piątkowski J, Kościelniak D, Gregorczyk-Maga I, Kołodziej I et al. Effect of histatin-5 and lysozyme on the ability of Streptococcus mutans to form biofilms in vitro conditions. Postepy Hig Med Dosw. 2015;69.

109. He J, Yarbrough DK, Kreth J, Anderson MH, Shi W, Eckert R. Systematic approach to optimizing specifically targeted antimicrobial peptides against Streptococcus mutans. Antimicrob Agents Chemother. 2010;54(5):2143-51. doi:https://doi.org/10.1128/AAC.01391-09.

110. Lundy FT, O'Hare MM, McKibben BM, Fulton CR, Briggs JE, Linden GJ. Radioimmunoassay quantification of adrenomedullin in human gingival crevicular fluid. Arch Oral Biol. 2006;51(4):334-8. doi:https://doi.org/10.1016/j.archoralbio.2005.08.006.

111. Elad S, Epstein JB, von Bültzingslöwen I, Drucker S, Tzach R, Yarom N. Topical immunomodulators for management of oral mucosal conditions, a systematic review; Part II: miscellaneous agents. Expert Opin Emerg Drugs. 2011;16(1):183-202. doi:https://doi.org/10.1517/14728214.2011.528390.

112. Jungell P, Malmström M. Cyclosporin A mouthwash in the treatment of oral lichen planus. Int J Oral Maxillofac Surg. 1996;25(1):60-2. doi:https://doi.org/10.1016/S0901-5027(96)80014-2.

113. Eisen D, Ellis CN. Topical cyclosporine for oral mucosal disorders. Journal of the American Academy of Dermatology. 1990;23(6):1259-64. doi:https://doi.org/10.1016/0190-9622(90)70352-I.

114. Voûte AB, Schulten EA, Langendijk PN, Nieboer C, van der Waal I. Cyclosporin A in an adhesive base for treatment of recalcitrant oral lichen planus: an open trial. Oral Surg Oral Med Oral Pathol. 1994;78(4):437-41. doi:https://doi.org/10.1016/0030-4220(94)90034-5.

115. Thongprasom K, Chaimusig M, Korkij W, Sererat T, Luangjarmekorn L, Rojwattanasirivej S. A randomized-controlled trial to compare topical cyclosporin with triamcinolone acetonide for the treatment of oral lichen planus. J Oral Pathol Med. 2007;36(3):142-6. doi:https://doi.org/10.1111/j.16000714.2007.00510.x. 
116. Elad S, Epstein JB, Yarom N, Drucker S, Tzach R, Bültzingslöwen Iv. Topical immunomodulators for management of oral mucosal conditions, a systematic review; part I: calcineurin inhibitors. Expert Opin Emerg Drugs. 2010;15(4):713-26. doi:https://doi.org/10.1517/14728214.2010.528389.

117. Conrotto D, Carbone M, Carrozzo M, Arduino P, Broccoletti R, Pentenero M et al. Ciclosporin vs. clobetasol in the topical management of atrophic and erosive oral lichen planus: a double-blind, randomized controlled trial. Br J Dermatol. 2006;154(1):139-45. doi:https://doi.org/10.1111/j.13652133.2005.06920.x.

118. Atridox® - Den-Mat Holdings, LLC. DailyMed. 2020. https://dailymed.nlm.nih.gov/dailymed/fda/fdaDrugXsl.cfm?setid=2550dbc0-8bdd-4bca-81d5eec77ee6fbd5\&type=display. Accessed 4 Jan 2021.

119. Chi M, Qi M, Wang P, Weir MD, Melo MA, Sun X et al. Novel Bioactive and therapeutic dental polymeric materials to inhibit periodontal pathogens and biofilms. Int J Mol Sci. 2019;20(2):278.

120. Lboutounne H. Dental medicine nanosystems: nanoparticles and their use in dentistry and oral health care. Int J Dent Oral Health. 2017;3(10):145-57. doi:https://doi.org/10.25141/2471-657X-2017-10.0150.

121. Benoit DS, Sims Jr KR, Fraser D. Nanoparticles for oral biofilm treatments. ACS nano. 2019;13(5):4869-75. doi:https://doi.org/10.1021/acsnano.9b02816.

122. Noronha VT, Paula AJ, Duran G, Galembeck A, Cogo-Mueller K, Franz-Montan M et al. Silver nanoparticles in dentistry. Dental Materials. 2017;33(10):111026. doi:https://doi.org/10.1016/j.dental.2017.07.002.

123. Bapat RA, Chaubal TV, Joshi CP, Bapat PR, Choudhury H, Pandey M et al. An overview of application of silver nanoparticles for biomaterials in dentistry. Mater Sci Eng C. 2018;91:881-98. doi:https://doi.org/10.1016/j.msec.2018.05.069.

124. Nguyen S, Hiorth M. Advanced drug delivery systems for local treatment of the oral cavity. Ther delivery. 2015;6(5):595-608. doi:https://doi.org/10.4155/tde.15.5.

125. Pokrowiecki R, Wojnarowicz J, Zareba T, Koltsov I, Lojkowski W, Tyski S et al. Nanoparticles And Human Saliva: A Step Towards Drug Delivery Systems For Dental And Craniofacial Biomaterials. Int J Nanomedicine. 2019;14:9235. doi:https://doi.org/10.2147/IJN.S221608.

126. Corrêa JM, Mori M, Sanches HL, Cruz ADd, Poiate E, Poiate IAVP. Silver nanoparticles in dental biomaterials. Int J Biomater. $2015 ; 2015$. doi:https://doi.org/10.1155/2015/485275.

127. Schmalz G, Hickel R, Van Landuyt KL, Reichl FX. Scientific update on nanoparticles in dentistry. Int Dent J. 2018;68(5):299-305. doi:https://doi.org/10.1111/idj.12394|.

128. Leung KC-F, Seneviratne CJ, Li X, Leung PC, Lau CBS, Wong C-H et al. Synergistic antibacterial effects of nanoparticles encapsulated with Scutellaria baicalensis and pure chlorhexidine on oral bacterial biofilms. Nanomaterials. 2016;6(4):61. doi:https://doi.org/10.3390/nano6040061.

129. Sadony DM, Abozaid HE-s. Antibacterial effect of metallic nanoparticles on Streptococcus mutans bacterial strain with or without diode laser (970 nm). Bull Natl Res Cent. 2020;44(1):2. doi:https://doi.org/10.1186/s42269-019-0262-z.

130. Targino AGR, Flores MAP, dos Santos Junior VE, Bezerra FdGB, de Luna Freire H, Galembeck A et al. An innovative approach to treating dental decay in children. A new anti-caries agent. J Mater Sci Mater Med. 2014;25(8):2041-7. doi:https://doi.org/10.1007/s10856-014-5221-5.

131. Song W, Ge S. Application of antimicrobial nanoparticles in dentistry. Molecules. 2019;24(6):1033. doi:https://doi.org/10.3390/molecules24061033.

132. Vega-Jiménez A, Almaguer-Flores A, Flores-Castañeda M, Camps E, Uribe-Ramírez M, Aztatzi-Aguilar $O$ et al. Bismuth subsalicylate nanoparticles with anaerobic antibacterial activity for dental applications. Nanotechnology. 2017;28(43):435101. doi:https://doi.org/10.1088/1361-6528/aa8838.

133. Cousins B, Allison H, Doherty P, Edwards C, Garvey M, Martin D et al. Effects of a nanoparticulate silica substrate on cell attachment of Candida albicans. J Appl Microbiol. 2007;102(3):757-65. doi:https://doi.org/10.1111/j.1365-2672.2006.03124.x.

134. Wang Y, Zhao Q, Han N, Bai L, Li J, Liu J et al. Mesoporous silica nanoparticles in drug delivery and biomedical applications. Nanomed Nanotechnol Biol Med. 2015;11(2):313-27.

135. Seneviratne CJ, Leung KC-F, Wong C-H, Lee S-F, Li X, Leung PC et al. Nanoparticle-encapsulated chlorhexidine against oral bacterial biofilms. PLoS One. 2014;9(8):e103234. doi:https://doi.org/10.1371/journal.pone.0103234.

136. He J, Zhu X, Qi Z, Wang C, Mao X, Zhu C et al. Killing dental pathogens using antibacterial graphene oxide. ACS Appl Mater Interfaces. 2015;7(9):5605-11. doi:https://doi.org/10.1021/acsami.5b01069.

137. Xie H, Cao T, Rodríguez-Lozano FJ, Luong-Van EK, Rosa V. Graphene for the development of the next-generation of biocomposites for dental and medical applications. Dent Mater. 2017;33(7):765-74. doi:https://doi.org/10.1016/j.dental.2017.04.008.

138. Daniyal M, Liu B, Wang W. Comprehensive review on graphene oxide for use in drug delivery system. Curr Med Chem. 2020. doi:https://doi.org/10.2174/13816128256661902011296290.

139. Nagata JY, Hioka N, Kimura E, Batistela VR, Terada RSS, Graciano AX et al. Antibacterial photodynamic therapy for dental caries: evaluation of the photosensitizers used and light source properties. Photodiagn Photodyn Ther. 2012;9(2):122-31. doi:https://doi.org/10.1016/j.pdpdt.2011.11.006.

140. Wood S, Metcalf D, Devine D, Robinson C. Erythrosine is a potential photosensitizer for the photodynamic therapy of oral plaque biofilms. $J$ Antimicrob Chemother. 2006;57(4):680-4. doi:https://doi.org/10.1093/jac/dkl021.

141. Maisch T, Wagner J, Papastamou V, Nerl HJ, Hiller KA, Szeimies RM et al. Combination of 10\% EDTA, photosan, and a blue light hand-held photopolymerizer to inactivate leading oral bacteria in dentistry in vitro. J Appl Microbiol. 2009;107(5):1569-78. doi:https://doi.org/10.1111/j.13652672.2009.04342.x.

142. Pileggi G, Wataha JC, Girard M, Grad I, Schrenzel J, Lange N et al. Blue light-mediated inactivation of Enterococcus faecalis in vitro. Photodiagn Photodyn Ther. 2013;10(2):134-40. doi:https://doi.org/10.1016/j.pdpdt.2012.11.002.

143. Perni S, Piccirillo C, Pratten J, Prokopovich P, Chrzanowski W, Parkin IP et al. The antimicrobial properties of light-activated polymers containing methylene blue and gold nanoparticles. Biomaterials. 2009;30(1):89-93. doi:https://doi.org/10.1016/j.biomaterials.2008.09.020.

Page 26/31 
144. Qi M, Chi M, Sun X, Xie X, Weir MD, Oates TW et al. Novel nanomaterial-based antibacterial photodynamic therapies to combat oral bacterial biofilms and infectious diseases. Int J Nanomedicine. 2019;14:6937. doi:https://doi.org/10.2147/IJN.S212807.

145. Rad MR, Pourhajibagher M, Rokn AR, Barikani HR, Bahador A. Effect of Antimicrobial Photodynamic Therapy Using Indocyanine Green Doped with Chitosan Nanoparticles on Biofilm Formation-Related Gene Expression of Aggregatibacter actinomycetemcomitans. Front Dent. 2019;16(3):187. doi:https://doi.org/10.18502/fid.v16i3.1590.

146. Nagahara A, Mitani A, Fukuda M, Yamamoto H, Tahara K, Morita I et al. Antimicrobial photodynamic therapy using a diode laser with a potential new photosensitizer, indocyanine green-loaded nanospheres, may be effective for the clearance of P orphyromonas gingivalis. J Periodontal Res. 2013;48(5):591-9. doi:https://doi.org/10.1111/jre.12042.

147. De Freitas LM, Calixto GMF, Chorilli M, Giusti JSM, Bagnato VS, Soukos NS et al. Polymeric nanoparticle-based photodynamic therapy for chronic periodontitis in vivo. Int J Mol Sci. 2016;17(5):769. doi:https://doi.org/10.3390/ijms17050769.

148. Rizvi SA, Saleh AM. Applications of nanoparticle systems in drug delivery technology. Saudi Pharm J. 2018;26(1):64-70.

149. Costa EM, Silva S, Veiga M, Tavaria FK, Pintado MM. A review of chitosan's effect on oral biofilms: perspectives from the tube to the mouth. J Oral Biosci. 2017;59(4):205-10. doi:https://doi.org/10.1016/j.job.2017.07.001.

150. Aithal GC, Nayak UY, Mehta C, Narayan R, Gopalkrishna P, Pandiyan S et al. Localized in situ nanoemulgel drug delivery system of quercetin for periodontitis: development and computational simulations. Molecules. 2018;23(6):1363. doi:https://doi.org/10.3390/molecules23061363.

151. Madi M, Pavlic V, Samy W, Alagl A. The anti-inflammatory effect of locally delivered nano-doxycycline gel in therapy of chronic periodontitis. Acta Odontol Scand. 2018;76(1):71-6. doi:https://doi.org/10.1080/00016357.2017.1385096.

152. Beg S, Dhiman S, Sharma T, Jain A, Sharma RK, Jain A et al. Stimuli Responsive In Situ Gelling Systems Loaded with PLGA Nanoparticles of Moxifloxacin Hydrochloride for Effective Treatment of Periodontitis. AAPS PharmSciTech. 2020;21(3):76. doi:https://doi.org/10.1208/s12249-019-1613-7.

153. Álvarez AL, Espinar FO, Méndez JB. The application of microencapsulation techniques in the treatment of endodontic and periodontal diseases. Pharmaceutics. 2011;3(3):538-71. doi:https://doi.org/10.3390/pharmaceutics3030538.

154. Kmiec M, Pighinelli L, Tedesco M, Silva M, Reis V. Chitosan-properties and applications in dentistry. Adv Tissue Eng Regen Med. $2017 ; 2(4): 00035$. doi:https://doi.org/10.15406/atroa.2017.02.00035.

155. Virlan MJR, Miricescu D, Totan A, Greabu M, Tanase C, Sabliov CM et al. Current uses of poly (lactic-co-glycolic acid) in the dental field: a comprehensive review. J Chem. 2015;2015. doi:https://doi.org/10.1155/2015/525832.

156. Kawakita ER, Ré ACS, Peixoto MPG, Ferreira MP, Ricomini-Filho AP, Freitas 0 et al. Effect of Chitosan Dispersion and Microparticles on Older Streptococcus mutans Biofilms. Molecules. 2019;24(9):1808. doi:https://doi.org/10.3390/molecules24091808.

157. Moura LA, Ribeiro FV, Aiello TB, Duek EADR, Sallum EA, Nociti Junior FH et al. Characterization of the release profile of doxycycline by PLGA microspheres adjunct to non-surgical periodontal therapy. J Biomater Sci Polym Ed. 2015;26(10):573-84. doi:https://doi.org/10.1080/09205063.2015.1045249.

158. Arestin® - OroPharma. 2020. https://www.arestin.com/. Accessed 4 Jan 2021.

159. Dixit R, Puthli S. Oral strip technology: overview and future potential. J Control Release. 2009;139(2):94-107. doi:https://doi.org/10.1016/j.jconrel.2009.06.014.

160. Joshi D, Garg T, Goyal AK, Rath G. Advanced drug delivery approaches against periodontitis. Drug Deliv. 2016;23(2):363-77. doi:https://doi.org/10.3109/10717544.2014.935531.

161. Rajeshwari H, Dhamecha D, Jagwani S, Rao M, Jadhav K, Shaikh S et al. Local drug delivery systems in the management of periodontitis: A scientific review. J Control Release. 2019;307:393-409. doi:https://doi.org/10.1016/j.jconrel.2019.06.038.

162. Friesen LR, Williams KB, Krause LS, Killoy WJ. Controlled local delivery of tetracycline with polymer strips in the treatment of periodontitis. J Periodontol. 2002;73(1):13-9. doi:https://doi.org/10.1902/jop.2002.73.1.13.

163. Mahajania M, Laddha R, Shelke A, Gadhiya N, Narkhede S, Shetty G. Effect of Subgingival Doxycycline Placement on Clinical and Microbiological Parameters in Inflammatory Periodontal Disease: Both in Vivo and in Vitro Studies. J Contemp Dent Pract. 2018;19(10):1228-34.

164. Deasy P, Collins AE, Maccarthy DJ, Russell R. Use of strips containing tetracycline hydrochloride or metronidazole for the treatment of advanced periodontal disease. J Pharm Pharmacol. 1989;41(10):694-9. doi:https://doi.org/10.1111/j.2042-7158.1989.tb06343.x.

165. Goodson J, Cugini M, Kent R, Armitage G, Cobb C, Fine D et al. Multicenter evaluation of tetracycline fiber therapy: II. Clinical response. J Periodontal Res. 1991;26(4):371-9. doi:https://doi.org/10.1111/j.1600-0765.1991.tb02076.x.

166. Steinberg D, Friedman M, Soskolne A, Sela M. A new degradable controlled release device for treatment of periodontal disease: in vitro release study. J Periodontol. 1990;61(7):393-8. doi:https://doi.org/10.1902/jop.1990.61.7.393.

167. Meireles AB, Corrêa DK, da Silveira JV, Millás AL, Bittencourt E, de Brito-Melo GE et al. Trends in polymeric electrospun fibers and their use as oral biomaterials. Exp Biol Med. 2018;243(8):665-76. doi:https://doi.org/10.1177/1535370218770404.

168. Steinberg D, Friedman M. Sustained-release delivery of antimicrobial drugs for the treatment of periodontal diseases: Fantasy or already reality? Periodontology. 2020;84(1):176-87. doi:https://doi.org/10.1111/prd.12341.

169. Chou S-F, Carson D, Woodrow KA. Current strategies for sustaining drug release from electrospun nanofibers. J Control Release. $2015 ; 220: 584-91$. doi:https://doi.org/10.1016/j.jconrel.2015.09.008.

170. dos Santos DM, Chagas PA, Leite IS, Inada NM, de Annunzio SR, Fontana CR et al. Core-sheath nanostructured chitosan-based nonwovens as a potential drug delivery system for periodontitis treatment. Int J Biol Macromol. 2020;142:521-34. doi:https://doi.org/10.1016/j.ijbiomac.2019.09.124.

171. Mahmoud MY, Sapare S, Curry KC, Demuth DR, Steinbach-Rankins JM. Rapid release polymeric fibers for inhibition of Porphyromonas gingivalis adherence to Streptococcus gordonii. Front Chem. 2020;7:926. doi:https://doi.org/10.3389/fchem.2019.00926.

Page 27/31 
172. Lim SY, Dafydd M, Ong J, Ord-McDermott LA, Board-Davies E, Sands K et al. Mucoadhesive thin films for the simultaneous delivery of microbicide and anti-inflammatory drugs in the treatment of periodontal diseases. Int J Pharm. 2020;573:118860. doi:https://doi.org/10.1016/j.ijpharm.2019.118860.

173. Barat R, Srinatha A, Pandit JK, Anupurba S, Mittal N. Chitosan inserts for periodontitis: influence of drug loading, plasticizer and crosslinking on in vitro metronidazole release. Acta Pharm. 2007;57(4):469-77. doi:https://doi.org/10.2478/v10007-007-0037-1

174. Karki S, Kim H, Na S-J, Shin D, Jo K, Lee J. Thin films as an emerging platform for drug delivery. Asian J Pharm Sci. 2016;11(5):559-74. doi:https://doi.org/10.1016/j.ajps.2016.05.004.

175. Atac M, Şenel S, Eren A. Application of chitosan films in sulcoplasty operations. Adv Chitin Sci. 2005;4:270-4.

176. Alves TF, Rios AC, da Silva Pontes K, Portella DL, Aranha N, Severino P et al. Bilayer mucoadhesive buccal film for mucosal ulcers treatment: development, characterization, and single study case. Pharmaceutics. 2020;12(7):657. doi:https://doi.org/10.3390/pharmaceutics12070657.

177. Costa JSR, de Oliveira Cruvinel K, Oliveira-Nascimento L. A mini-review on drug delivery through wafer technology: Formulation and manufacturing of buccal and oral lyophilizates. J Adv Res. 2019;20:33-41. doi:https://doi.org/10.1016/j.jare.2019.04.010.

178. Timur SS, Yüksel S, Akca G, Şenel S. Localized drug delivery with mono and bilayered mucoadhesive films and wafers for oral mucosal infections. Int $J$ Pharm. 2019;559:102-12. doi:https://doi.org/10.1016/j.ijpharm.2019.01.029.

179. Schkarpetkin D, Reise M, Wyrwa R, Völpel A, Berg A, Schweder M et al. Development of novel electrospun dual-drug fiber mats loaded with a combination of ampicillin and metronidazole. Dent Mater. 2016;32(8):951-60. doi:https://doi.org/10.1016/j.dental.2016.05.002.

180. Uraz A, Boynueğri D, Özcan G, Karaduman B, Uc D, Şenel S et al. Two percent chitosan mouthwash: A microbiological and clinical comparative study. J Dent Sci. 2012;7(4):342-9. doi:https://doi.org/10.1016/j.jds.2012.05.003.

181. Beyth N, Redlich M, Harari D, Friedman M, Steinberg D. Effect of sustained-release chlorhexidine varnish on Streptococcus mutans and Actinomyces viscosus in orthodontic patients. Am J Orthod Dentofac Orthop. 2003;123(3):345-8. doi:https://doi.org/10.1067/mod.2003.19.

182. Jentsch H, Eckert FR, Eschrich K, Stratul SI, Kneist S. Antibacterial action of chlorhexidine/thymol containing varnishes in vitro and in vivo. Int J Dent Hyg. 2014;12(3):168-73. doi:https://doi.org/10.1111/idh.12079.

183. Reise M, Wyrwa R, Muller U, Zylinski M, Volpel A, Schnabelrauch M et al. Release of metronidazole from electrospun poly(L-lactide-co-D/L-lactide) fibers for local periodontitis treatment. Dent Mater. 2012;28(2):179-88. doi:https://doi.org/10.1016/j.dental.2011.12.006.

184. Khan G, Yadav SK, Patel RR, Nath G, Bansal M, Mishra B. Development and evaluation of biodegradable chitosan films of metronidazole and levofloxacin for the management of periodontitis. Aaps Pharmscitech 2016;17(6):1312-25. doi:https://doi.org/10.1208/s12249-015-0466-y.

185. Leung WK, Jin L, Yau JY, Sun Q, Corbet EF. Microflora cultivable from minocycline strips placed in persisting periodontal pockets. Arch Oral Biol. 2005;50(1):39-48. doi:https://doi.org/10.1016/j.archoralbio.2004.08.002.

186. Swain GP, Patel S, Gandhi J, Shah P. Development of Moxifloxacin Hydrochloride loaded in-situ gel for the treatment of periodontitis: In-vitro drug release study and antibacterial activity. J Oral Biol Craniofac Res 2019;9(3):190-200. doi:https://doi.org/10.1016/j.jobcr.2019.04.001.

187. Ranjbar-Mohammadi M, Zamani M, Prabhakaran M, Bahrami SH, Ramakrishna S. Electrospinning of PLGA/gum tragacanth nanofibers containing tetracycline hydrochloride for periodontal regeneration. Mater Sci Eng C. 2016;58:521-31. doi:https://doi.org/10.1016/j.msec.2015.08.066.

188. Bottino MC, Münchow EA, Albuquerque MT, Kamocki K, Shahi R, Gregory RL et al. Tetracycline-incorporated polymer nanofibers as a potential dental implant surface modifier. J Biomed Mater Res B. 2017;105(7):2085-92. doi:https://doi.org/10.1002/jbm.b.33743.

189. Xu X, Gu Z, Chen X, Shi C, Liu C, Liu M et al. An injectable and thermosensitive hydrogel: Promoting periodontal regeneration by controlled-release of aspirin and erythropoietin. Acta biomater. 2019;86:235-46. doi:https://doi.org/10.1016/j.actbio.2019.01.001.

190. Ashri LY, Amal El Sayeh F, Ibrahim MA, Alshora DH. Optimization and evaluation of chitosan buccal films containing tenoxicam for treating chronic periodontitis: In vitro and in vivo studies. Journal of Drug Delivery Science and Technology 2020:101720.

191. Özdoğan Al, Akca G, Şenel S. Development and in vitro evaluation of gel formulation of atorvastatin solid dispersions. J Drug Deliv Sci Tec. $2020: 102199$. doi:https://doi.org/10.1016/j.jddst.2020.102199.

192. Hosseinzadeh S, Hamedi S, Esmaeili E, Kabiri M, Babaie A, Soleimani M et al. Mucoadhesive nanofibrous membrane with anti-inflammatory activity. Polym Bull. 2019;76(9):4827-40. doi:https://doi.org/10.1007/s00289-018-2618-1.

193. Chow EP, Howden BP, Walker S, Lee D, Bradshaw CS, Chen MY et al. Antiseptic mouthwash against pharyngeal Neisseria gonorrhoeae: a randomised controlled trial and an in vitro study. Sex Transm Infect. 2017;93(2):88-93. doi:http://dx.doi.org/10.1136/sextrans-2016-052753.

194. de Toledo LdAS, Rosseto HC, Dos Santos RS, Spizzo F, Del Bianco L, Montanha MC et al. Thermal magnetic field activated propolis release from liquid crystalline system based on magnetic nanoparticles. AAPS PharmSciTech. 2018;19(7):3258-71. doi:https://doi.org/10.1208/s12249-018-1163-4.

195. Hirasawa M, Takada K, Makimura M, Otake S. Improvement of periodontal status by green tea catechin using a local delivery system: a clinical pilot study. J Periodontal Res. 2002;37(6):433-8. doi:https://doi.org/10.1034/j.1600-0765.2002.01640.x.

196. Gottumukkala SN, Sudarshan S, Mantena SR. Comparative evaluation of the efficacy of two controlled release devices: Chlorhexidine chips and indigenous curcumin based collagen as local drug delivery systems. Contemp Clin Dent. 2014;5(2):175. doi:https://doi.org/10.4103/0976-237X.132310.

197. Zupančič Š, Baumgartner S, Lavrič Z, Petelin M, Kristl J, Technology. Local delivery of resveratrol using polycaprolactone nanofibers for treatment of periodontal disease. J Drug Deliv Sci Tec. 2015;30:408-16. doi:https://doi.org/10.1016/j.jddst.2015.07.009.

198. Watanabe S, Suemaru K, Takechi K, Kaji H, Imai K, Araki H. Oral mucosal adhesive films containing royal jelly accelerate recovery from 5-fluorouracilinduced oral mucositis. J Pharmacol Sci. 2013:12181FP. doi:https://doi.org/10.1254/jphs.12181FP.

199. Khajuria DK, Patil ON, Karasik D, Razdan R. Development and evaluation of novel biodegradable chitosan based metformin intrapocket dental film for the management of periodontitis and alveolar bone loss in a rat model. Arch Oral Biol. 2018;85:120-9. doi:https://doi.org/10.1016/j.archoralbio.2017.10.009. 
200. Saha S, Tomaro-Duchesneau C, Daoud JT, Tabrizian M, Prakash S. Novel probiotic dissolvable carboxymethyl cellulose films as oral health biotherapeutics: in vitro preparation and characterization. Expert Opin Drug Deliv. 2013;10(11):1471-82. doi:https://doi.org/10.1517/17425247.2013.799135.

201. Sánchez M, Toledano-Osorio M, Bueno J, Figuero E, Toledano M, Medina-Castillo A et al. Antibacterial effects of polymeric PolymP-n Active nanoparticles. An in vitro biofilm study. Dent Mater. 2019;35(1):156-68. doi:https://doi.org/10.1016/j.dental.2018.11.015.

202. Di Turi G, Riggio C, Vittorio O, Marconcini S, Briguglio F, Funel N et al. Sub-Micrometric Liposomes as Drug Delivery Systems in the Treatment and Periodontitis. Int J Immunopathol Pharmacol 2012;25(3):657-70. doi:https://doi.org/10.1177/039463201202500312.

203. He Y, Jin Y, Wang X, Yao S, Li Y, Wu Q et al. An antimicrobial peptide-loaded gelatin/chitosan nanofibrous membrane fabricated by sequential layer-bylayer electrospinning and electrospraying techniques. Nanomaterials. 2018;8(5):327. doi:https://doi.org/10.3390/nano8050327.

204. Chaudhary B, Verma S. Preparation and evaluation of novel in situ gels containing acyclovir for the treatment of oral herpes simplex virus infections. Sci World J. 2014;2014. doi:https://doi.org/10.1155/2014/280928.

205. Al-Dhubiab BE, Nair AB, Kumria R, Attimarad M, Harsha S. Formulation and evaluation of nano based drug delivery system for the buccal delivery of acyclovir. Colloids Surf B Biointerfaces. 2015;136:878-84. doi:https://doi.org/10.1016/j.colsurfb.2015.10.045.

206. Downing C, Moayyad J, Tamirisa A, Tyring S. Acyclovir Lauriad®: a muco-adhesive buccal tablet for the treatment of recurrent herpes labialis. Expert Rev Anti Infect Ther. 2014;12(3):283-7. doi:https://doi.org/10.1586/14787210.2014.880337.

207. Lin L, Chen X, Cui P, Wang J, Guo Z, Lu N et al. Topical application of penciclovir cream for the treatment of herpes simplex facialis/labialis: a randomized, double-blind, multicentre, aciclovir-controlled trial. J Dermatol Treat. 2002;13(2):67-72. doi:https://doi.org/10.1080/095466302317584412.

208. Moura MDG, Haddad JPA, Senna MIB, e Ferreira EF, Mesquita RA. A new topical treatment protocol for oral hairy leukoplakia. Oral Surg Oral Med Oral Pathol Oral Radiol Endod. 2010;110(5):611-7. doi:https://doi.org/10.1016/j.tripleo.2010.05.015.

209. Cui Z, Mumper RJ. Bilayer films for mucosal (genetic) immunization via the buccal route in rabbits. Pharm Res. 2002;19(7):947-53. doi:https://doi.org/10.1023/A:1016454003450.

210. Gajdošová M, Vetchý D, Muselík J, Gajdziok J, Juřica J, Vetchá M et al. Bilayer mucoadhesive buccal films with prolonged release of ciclopirox olamine for the treatment of oral candidiasis: In vitro development, ex vivo permeation testing, pharmacokinetic and efficacy study in rabbits. Int $\mathrm{J}$ Pharm. 2020:120086. doi:https://doi.org/10.1016/j.ijpharm.2020.120086.

211. Harish N, Prabhu P, Charyulu R, Gulzar M, Subrahmanyam E. Formulation and evaluation of in situ gels containing clotrimazole for oral candidiasis. Indian J Pharm Sci. 2009;71(4):421.

212. Rençber S, Karavana SY, Yilmaz FF, Eraç B, Nenni M, Gurer-Orhan H et al. Formulation and evaluation of fluconazole loaded oral strips for local treatment of oral candidiasis. J Drug Deliv Sci Tec. 2019;49:615-21. doi:https://doi.org/10.1016/j.jddst.2018.12.035.

213. Mendes A, Silva A, Catita J, Cerqueira F, Gabriel C, Lopes CM. Miconazole-loaded nanostructured lipid carriers (NLC) for local delivery to the oral mucosa: improving antifungal activity. Colloids Surf B Biointerfaces. 2013;111:755-63. doi:https://doi.org/10.1016/j.colsurfb.2013.05.041.

214. Vazquez JA, Patton LL, Epstein JB, Ramlachan P, Mitha I, Noveljic Z et al. Randomized, comparative, double-blind, double-dummy, multicenter trial of miconazole buccal tablet and clotrimazole troches for the treatment of oropharyngeal candidiasis: study of miconazole Lauriad ${ }^{\circledR}$ efficacy and safety (SMiLES). HIV Clin Trials. 2010;11(4):186-96. doi:https://doi.org/10.1310/hct1104-186.

215. Uzunoğlu B, Wilson CG, Sağıroğlu M, Yüksel S, Şenel S. Mucoadhesive bilayered buccal platform for antifungal drug delivery into the oral cavity. Drug Deliv Transl Res. 2020:1-10. doi:https://doi.org/10.1007/s13346-020-00798-1.

216. Roque L, Alopaeus J, Reis C, Rijo P, Molpeceres J, Hagesaether E et al. Mucoadhesive assessment of different antifungal nanoformulations. Bioinspir Biomim. 2018;13(5):055001. doi:https://doi.org/10.1088/1748-3190/aad488.

217. Martin MJ, Calpena AC, Fernandez F, Mallandrich M, Gálvez P, Clares B. Development of alginate microspheres as nystatin carriers for oral mucosa drug delivery. Carbohyd Polym. 2015;117:140-9. doi:https://doi.org/10.1016/j.carbpol.2014.09.032.

218. Soriano-Ruiz JL, Calpena-Capmany AC, Cañadas-Enrich C, Bozal-de Febrer N, Suñer-Carbó J, Souto EB et al. Biopharmaceutical profile of a clotrimazole nanoemulsion: Evaluation on skin and mucosae as anticandidal agent. Int J Pharm. 2019;554:105-15. doi:https://doi.org/10.1016/j.ijpharm.2018.11.002.

219. Szekalska M, Wróblewska M, Trofimiuk M, Basa A, Winnicka K. Alginate Oligosaccharides Affect Mechanical Properties and Antifungal Activity of Alginate Buccal Films with Posaconazole. Mar Drugs. 2019;17(12):692. doi:https://doi.org/10.3390/md17120692.

220. Serrano DR, Fernandez-Garcia R, Mele M, Healy AM, Lalatsa A. Designing fast-dissolving orodispersible films of amphotericin B for oropharyngeal candidiasis. Pharmaceutics. 2019;11(8):369. doi:https://doi.org/10.3390/pharmaceutics11080369.

221. Rai VK, Yadav NP, Sinha P, Mishra N, Luqman S, Dwivedi H et al. Development of cellulosic polymer based gel of novel ternary mixture of miconazole nitrate for buccal delivery. Carbohyd Polym. 2014;103:126-33. doi:https://doi.org/10.1016/j.carbpol.2013.12.019.

\section{Figures}




\section{Drug delivery for treatment of oral infections}

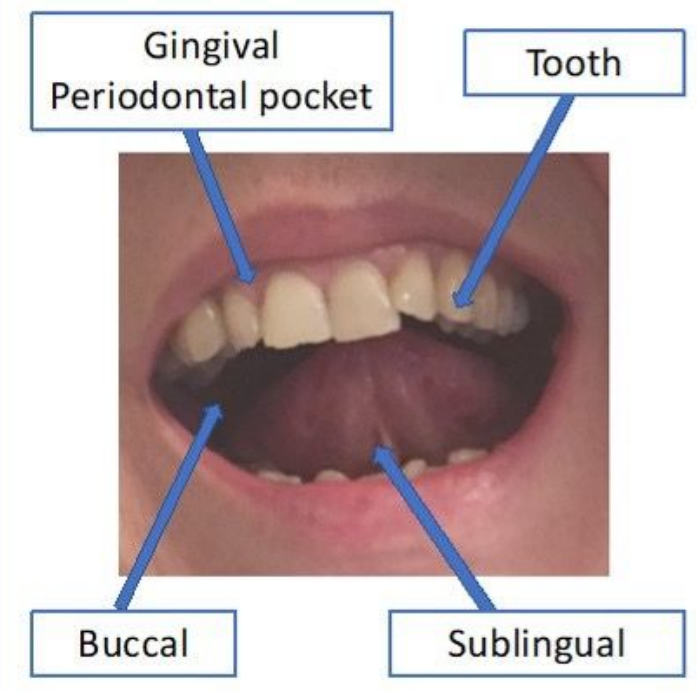

\section{Delivery systems}

Gels, Films, Tablets, Wafers, Fibers, Liposomes, Micelles, Polymeric Nanoparticles, Devices

\section{Therapeutics}

Antibiotics, Antifungals, Antivirals Antimicrobial peptides (AMPs), Antiinflammatory drugs, Immunomodulators, Antiseptics, Anesthetics, Enzyme inhibitors, Photactivateable agents/sensitizers

Figure 1

Drug groups and delivery systems for treatment of oral infections

Viruses
- Members of human
herpesvirus
HHV-1/HHV-2, HHV-3
(varicella-zoster virus),
HHV-4 (EBV), HHV-5
(CMV), HHV-8
- Human papillomavirus
(HPV)
- Measeles; Rubella
- Coxsackieviruses A and B
- Human
Immunodeficiency Virus
HIV-1 and HIV-2
EBV: Ebstein-Barr virüs
CVM: Cytomegalovirus

Bacteria
- Porphyromonas gingivalis,
Treponema denticola
- Mycobacterium
tuberculosis
- Mycobacterium leprae
- Neisseria gonorrhea
- Treponema pallidum
(Syphilis)
- Staphylococcus aureus
- Streptococci
- Fusobacterium sp. and
Spirochetes
- Polybacterial
Oral Biofilms
Bacteria in organized and
complex groups

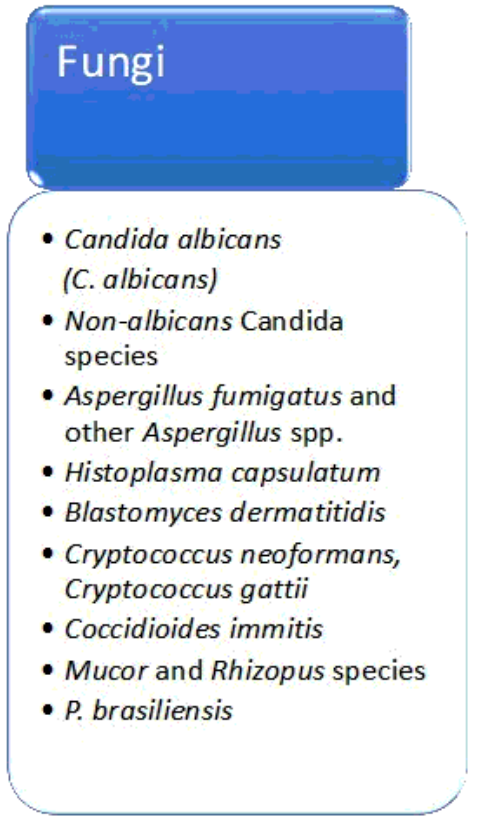

\section{ORAL MANIFESTATIONS}

Oral lesions (including ulcerative, erosive, or white lesions)

Swelling; Erythema; Mucosal pallor and atrophy;

Change in mucosal pigmentation; Periodontal bleeding and inflammation

Figure 2

Pathogens causing oral infections and resulting symptoms/manifestations 


\section{Supplementary Files}

This is a list of supplementary files associated with this preprint. Click to download.

- GraphicalAbstract.png 